\title{
Convergence of Discrete Period Matrices and Discrete Holomorphic Integrals for Ramified Coverings of the Riemann Sphere
}

\author{
Alexander I. Bobenko ${ }^{1}$. Ulrike Bücking ${ }^{2}$ (i) \\ Received: 3 September 2020 / Accepted: 1 June 2021 / Published online: 2 July 2021 \\ (C) The Author(s) 2021
}

\begin{abstract}
We consider the class of compact Riemann surfaces which are ramified coverings of the Riemann sphere $\hat{\mathbb{C}}$. Based on a triangulation of this covering we define discrete (multivalued) harmonic and holomorphic functions. We prove that the corresponding discrete period matrices converge to their continuous counterparts. In order to achieve an error estimate, which is linear in the maximal edge length of the triangles, we suitably adapt the triangulations in a neighborhood of every branch point. Finally, we also prove a convergence result for discrete holomorphic integrals for our adapted triangulations of the ramified covering.
\end{abstract}

Keywords Discrete analytic function - Riemann surface · Period matrix ·

Discrete holomorphic integral $\cdot$ Dirichlet energy $\cdot$ Approximation

Mathematics Subject Classification (2010) Primary 39A12 - 65M60 · 30E10;

Secondary $14 \mathrm{~K} 20 \cdot 30 \mathrm{~F} 30$

\section{Introduction}

Smooth holomorphic functions can be characterized in different ways. In particular, the real and imaginary part of any holomorphic function is harmonic and both are

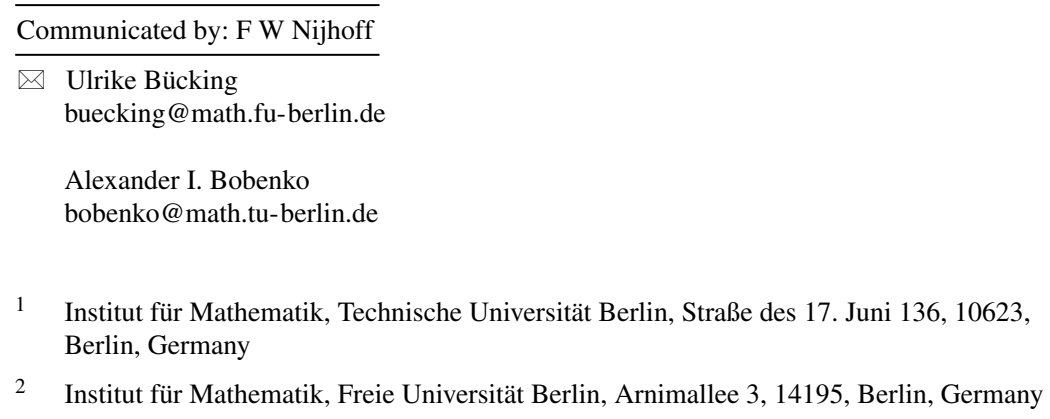


related by the Cauchy-Riemann equations. This perspective naturally led to linear discretizations of harmonic and holomorphic functions, starting with results for square grids, see [12, 14, 23]. Lelong-Ferrand further developed this theory of discrete harmonic and holomorphic functions in [19, 25]. MacNeal and Duffin generalized these notions in [15-17, 27]. In particular, they considered arbitrary triangulations in the plane and discovered the cotan-weights. The cotan-Laplacian is also considered for triangle meshes, for example for surfaces in discrete differential geometry, see [35], or for applications in computer graphics, see for example [32]. Further properties and theorems of the smooth theory of holomorphic functions have found recently discrete analogues in the discrete linear theory in [2, 3].

Note that there are other important nonlinear discretizations of holomorphic functions, for example involving circle packings or circle patterns $[8,9,36,38]$, connected to cross-ratios [6, 28], using discrete conformal equivalence $[1,10]$, or based on bicolored triangles $[18,34]$. The linear theory of holomorphic functions on rhombic lattices can be obtained as infinitesimal deformation of circle patterns [5].

Mercat generalized in [29] the discrete linear theory from planar subsets to discrete Riemann surfaces and introduced in [30, 31] discrete period matrices. In [4] numerical experiments are considered to compute discrete period matrices for polyhedral surfaces explicitly and compare them to known period matrices for the corresponding smooth surfaces. A convergence proof for the class of polyhedral surfaces was obtained in [7].

The interest in numerical computation of period matrices is for example motivated by the computation of finite-genus solutions of integrable differential equations. As Riemann surfaces may be represented as algebraic curves, this is often taken as a starting point for computing discrete period matrices. Recent results in this context include [13, 20-22, 33].

In this article, we take a different approach and consider Riemann surfaces which are ramified coverings of the Riemann sphere $\hat{\mathbb{C}}$. Based on a triangulation of this covering discrete period matrices can be obtained from this discrete data. Furthermore, we prove convergence of the discrete period matrices to their continuous counterparts (Theorem 3). In particular, we obtain an error estimate, which is linear in the maximal edge length of the triangles if we adapt the triangulations in a neighborhood of every branch point. The details of our 'adapted triangulations' will be explained in Section 2.3.

The convergence of discrete analytic functions to their continuous counterparts remains an important issue, although several results have been proved by now. In particular, for the linear theory, convergence was first shown for the square lattice [12, 25] and recently for more general quadrilateral lattices [7, 11, 37]. In this article, we prove the convergence of discrete holomorphic integrals (Abelian integrals of first kind) obtained from suitable triangulations of the ramified covering to their continuous counterparts (Theorem 4).

Our main results are stated in Section 2 and proved in Section 3. The proof is inspired by [7] and uses energy estimates which allow to prove the convergence of the discrete period matrices directly. Our results are also applied to improve the convergence results of [7] in Section 5. Finally, in Section 6, we present some numerical experiments. 


\section{Convergence Results for Discrete Period Matrices and Discrete Holomorphic Integrals for Ramified Coverings of $\hat{\mathbb{C}}$}

In the following, we consider any compact Riemann surface $\mathscr{R}$ of genus $g \geqslant 1$ which allows a branched covering map $f: \mathscr{R} \rightarrow \hat{\mathbb{C}}$. Using this covering map as a local chart, we always locally identify points in $\mathscr{R}$ with their images in $\hat{\mathbb{C}}$. Then for points in the complex plane $\mathbb{C}=\hat{\mathbb{C}} \backslash\{\infty\}$ we use the standard complex coordinate $z$. This map from $\mathscr{R}$ to $\mathbb{C}$ is denoted by $\operatorname{Pr}_{\mathscr{R}}$ and gives a local chart in a neighborhood about every point, except at branch points and infinity. For further use, we fix a radius $\rho>1$ such that the images of all branch points, except possibly $\infty$, have a distance at most $\rho / 2$ from the origin. In order to consider a neighborhood of infinity, we consider a second chart with the local coordinate $1 / z$. This map from $\mathscr{R}$ to $\mathbb{C}$ is denoted by $\operatorname{Pr}_{\mathscr{R}}^{\infty}$.

Let $T=T_{\mathscr{R}}$ be a triangulation of $\mathscr{R}$ such that all branch points are vertices. We assume that every triangle is contained in only one sheet of the covering. We will mostly consider this triangulation via its (local) image under the charts $\operatorname{Pr}_{\mathscr{R}}$ and $\operatorname{Pr}_{\mathscr{R}}^{\infty}$. In this sense, without further mention, we always identify this triangulation with the corresponding (multi-sheeted) triangulation on $\hat{\mathbb{C}}$ (which is the image $f(T)$ under the covering map) and with the (multi-sheeted) image of this triangulation of $\hat{\mathbb{C}}$ under the charts $\operatorname{Pr}_{\mathscr{R}}$ and $\operatorname{Pr}_{\mathscr{R}}^{\infty}$. We assume that this triangulation is a locally planar embedding in the complex plane $\mathbb{C}$ or equivalently in the Riemann sphere $\hat{\mathbb{C}}$, except at the branch points. From now on, we consider the vertices of the triangulation as points of $\mathbb{C}$, that is, we always apply the local charts $\operatorname{Pr}_{\mathscr{R}}$ and $\operatorname{Pr}_{\mathscr{R}}^{\infty}$. The edges connecting incident vertices will be straight line segments or circular arcs in $\mathbb{C}$, depending on the following distinction.

(i) All triangles whose images under the chart $\operatorname{Pr}_{\mathscr{R}}$ have at least two vertices in the open disc $B_{\rho}(0)$ of radius $\rho$ about the origin are geodesic, that is Euclidean triangles. We always consider these triangles to be embedded in $\mathbb{C}$.

(ii) All triangles whose image under the chart $\operatorname{Pr}_{\mathscr{R}}^{\infty}$ have all vertices contained in the closed disc $\bar{B}_{1 / \rho}(0)$ of radius $1 / \rho$ about the origin (that is, in the images under the chart $\operatorname{Pr}_{\mathscr{R}}$ all vertices are contained in the complement $\mathbb{C} \backslash B_{\rho}(0)$ ) are geodesic, that is Euclidean triangles. We always consider these triangles to be embedded in $\mathbb{C}$. Note that in the image under the chart $\operatorname{Pr}_{\mathscr{R}}$ these triangles are preimages of a geodesic triangulation with Euclidean triangles under the map $z \mapsto 1 / z$. Therefore, these triangles are in general bounded by circular arcs in the image under the chart $\operatorname{Pr} \mathscr{R}$.

(iii) The remaining triangles in the 'boundary region' are consequently in general bounded by two straight lines and one circular arc in the image under the chart $\operatorname{Pr} \mathscr{R}$. These triangles will be called boundary triangles and denoted by $F_{\rho}$. Finally, we assume that the edge lengths of all boundary triangles are strictly smaller than $\max \{\rho / 2,1\}$. As in the first case, we always consider these triangles under the chart $\operatorname{Pr}_{\mathscr{R}}$ to be embedded in $\mathbb{C}$. 
We denote by $V, E, \vec{E}, F$ the sets of vertices, edges, oriented edges, and faces of $T_{\mathscr{R}}$, respectively, and identify them locally with their images under the charts $\operatorname{Pr}_{\mathscr{R}}$ and $\operatorname{Pr}_{\mathscr{R}}^{\infty}$.

\subsection{Discrete Harmonic Functions}

We define weights on the edges $E$ of the triangulation $T_{\mathscr{R}}$ essentially by using cotanweights, but we distinguish two cases for edges $e=[x, y] \in E$ corresponding to the different cases above:

(i) If both triangles incident to $e$ are contained in the open disc $B_{\rho}(0)$ under the chart $\operatorname{Pr}_{\mathscr{R}}$ or in the closed disc $\bar{B}_{1 / \rho}(0)$ under the chart $\operatorname{Pr}_{\mathscr{R}}^{\infty}$, we use cotanweights

$$
c(e)=\frac{1}{2} \cot \alpha_{e}+\frac{1}{2} \cot \beta_{e},
$$

where $\alpha_{e}$ and $\beta_{e}$ are the angles opposite to the edge $e \in E$ in the two adjacent triangles, see Fig. 1.

(ii) If $e=[x, y]$ is incident to a boundary triangle in $F_{\rho}$, we use the chart $\operatorname{Pr}_{\mathscr{R}}$ and define the weight similarly as above as a sum $c(e)=C_{1}+C_{2}$ of two parts corresponding to the two incident triangles $\Delta_{1}, \Delta_{2}$. If there is a non-boundary triangle, say $\Delta_{1}$, incident to $e$, we consider the angle $\alpha_{e}$ in this triangle opposite to $e$ and set $C_{1}=\frac{1}{2} \cot \alpha_{e}$. The second part $C_{2}=C_{[x, y]}$ is defined below in (4) using a suitable interpolation function and the smooth Dirichlet energy. More details and explicit calculations are given in Appendix A.1.

Using our edge weights, we can define discrete harmonicity and a discrete Dirichlet energy for functions $u: V \rightarrow \mathbb{R}$ on the vertices of the triangulation $T_{\mathscr{R}}$. In particular, $u$ is called discrete harmonic if for every vertex $x \in V$ there holds

$$
\sum_{y \in V:[x, y] \in E} c([x, y])(u(y)-u(x))=0 .
$$

The energy of $u$ is

$$
E_{T}(u)=\sum_{e=[x, y] \in E} c([x, y])(u(y)-u(x))^{2} .
$$

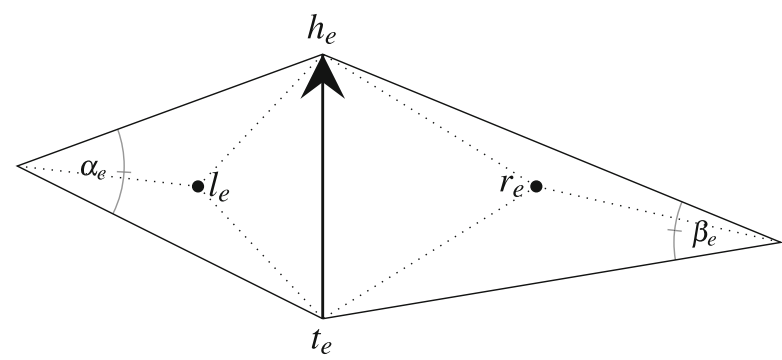

Fig. 1 Notation associated with an edge $e=\left[t_{e}, h_{e}\right] \in E$ and with its oriented version $\vec{e}=\overrightarrow{t_{e} h_{e}}$, see Sections 2.1 and 2.2 
The motivation for our choice of weights, in particular for the choice of weights for boundary triangles, is the following connection of discrete and smooth Dirichlet energies. Recall that for a continuous function on a compact Riemann surface $\mathscr{R}$ which is smooth almost everywhere the Dirichlet energy is defined as

$$
E(u)=\int_{\mathscr{R}}|\nabla u|^{2} .
$$

Then the discrete energy of a function $u: V \rightarrow \mathbb{R}$ is in fact the Dirichlet energy of the continuous interpolation function $I_{T} u$, defined piecewise on every triangle $\Delta[x, y, z]$ as follows:

(i) If at least two of the three vertices $x, y, z$ are contained in the open disc $B_{\rho}(0)$ under the chart $\operatorname{Pr}_{\mathscr{R}}$ or in the closed disc $\bar{B}_{1 / \rho}(0)$ under the chart $\operatorname{Pr}_{\mathscr{R}}^{\infty}$, we define $\left.I_{T} u\right|_{\Delta[x, y, z]}$ on this triangle as the linear interpolation of the values of $u$ at the vertices.

(ii) In the remaining case, $\Delta[x, y, z]$ is a boundary triangle in $F_{\rho}$ and under the chart $\operatorname{Pr}_{\mathscr{R}}$ there is exactly one vertex in $B_{\rho}(0)$, say $x$. We first define $I_{T} u$ on the boundary edges consistently with the definitions in (i). The two edges $[x, y]$ and $[x, z]$ are straight lines. On these edges we define $I_{T} u$ as the linear interpolation of the values of the vertices. On the arc $\widehat{y z}$ connecting $y$ and $z$ we use the corresponding transformed function $\tilde{u}=u \circ 1 / z$ and the interpolating function $\left.I_{T} u\right|_{\Delta[x, y, z]}=\widetilde{I_{T}} u_{\widetilde{\Delta}} \circ 1 / z$. Then for every straight line segment connecting $x$ to a point on the arc $\widehat{y z}$ we define $I_{T} u$ as the linear interpolation of the values on the endpoints.

Using this interpolation function, we obtain by elementary calculations (see Appendix A.1 for details) that

$$
\begin{aligned}
\int_{\Delta[x, y, z]}\left|\nabla I_{T} u\right|^{2}= & C_{[x, y]}(u(x)-u(y))^{2}+C_{[y, z]}(u(y)-u(z))^{2} \\
& +C_{[z, x]}(u(z)-u(x))^{2},
\end{aligned}
$$

where the constants $C_{[x, y]}, C_{[y, z]}, C_{[z, x]}$ only depend on the triangle $\Delta[x, y, z]$, see (13)-(15), and give one part of the weights associated to the edges $[x, y],[y, z],[z, x]$ respectively.

It is easy to see that for every triangulation of a ramified covering $\mathscr{R}$ of $\hat{\mathbb{C}}$ as above, $I_{T} u$ is a well-defined continuous function on $\mathscr{R}$. Furthermore, we have

Lemma 1 (Interpolation lemma)

$$
E\left(I_{T} u\right)=E_{T}(u) .
$$

Proof We can split the energy according to triangles $\Delta_{f}$ for $f \in F$.

$$
E\left(I_{T} u\right)=\sum_{\Delta_{f} \subset B_{\rho}(0)} \int_{\Delta_{f}}\left|\nabla I_{T} u\right|^{2}+\sum_{\Delta_{f} \subset \hat{\mathbb{C}} \backslash B_{\rho}(0)} \int_{\Delta_{f}}\left|\nabla I_{T} u\right|^{2}+\sum_{\Delta_{f} \in F_{\rho}(0)} \int_{\Delta_{f}}\left|\nabla I_{T} u\right|^{2}
$$

In particular, elementary calculations show that (4) holds for any triangle $\Delta[x, y, z]$. with suitable constants $C_{[x, y]}, C_{[y, z]}, C_{[z, x]}$ depending only on $\Delta[x, y, z]$. Duffin 
showed in [16, Section 4] that for Euclidean triangles these constants are one half of the cotan of the opposite angles. Using the conformal invariance of the Dirichlet integral for the triangles in $\hat{\mathbb{C}} \backslash B_{\rho}(0)$ and our choice of weights in the cases (i)-(ii) above, we obtain the claim.

Remark 1 It will be important to note that the constants $C_{[x, y]}, C_{[y, z]}, C_{[z, x]}$ defined by (4) are only small perturbations of the usual cotan-weights in the following sense. If the maximal edge length $h$ in the boundary triangle $\Delta[x, y, z] \in F_{\rho}$ is small enough and the angles in $\Delta[x, y, z]$ as well as the angles in the Euclidean triangle formed by the vertices $x, y, z$ lie in $[\delta, \pi-\delta]$ for some $\delta>0$, then elementary calculations and estimates show that

$$
\frac{1}{2} \cot \hat{\alpha}_{e}-C_{\delta, \rho} \cdot h \leqslant C_{e} \leqslant \frac{1}{2} \cot \hat{\alpha}_{e}+C_{\delta, \rho} \cdot h .
$$

for some constant $C_{\delta, \rho}>0$, where $\hat{\alpha}_{e}$ denotes the angle opposite to the edge $e$ in the Euclidean triangle formed by the vertices $x, y, z$. The details are given in Appendix A.1.

Note that the difference between the the angles in the Euclidean triangle with vertices $x, y, z$ and the corresponding angles in $\Delta[x, y, z]$ is of order $h$. Thus, for $h$ small enough, the corresponding estimates on $\left|C_{e}-\frac{1}{2} \cot \alpha_{e}\right|$ also hold for the actual angles $\alpha_{e}$ in the triangle $\Delta[x, y, z]$.

\subsection{Discrete Analytic Functions, Discrete Holomorphic Integrals and Discrete Period Matrices}

In the following, we define discrete analytic functions and discrete holomorphic integrals analogously as in [7].

For an oriented edge $\vec{e} \in \vec{E}$, we denote by $h_{e} \in V$ and $t_{e} \in V$ the head and the tail of $\vec{e}$, and by $l_{e} \in F$ and $r_{e} \in F$ the left shore and the right shore of $\vec{e}$, respectively, see Fig. 1. Two functions $u: V \rightarrow \mathbb{R}$ and $v: F \rightarrow \mathbb{R}$ are conjugate, if for each oriented edge $\vec{e} \in \vec{E}$ we have

$$
v\left(l_{e}\right)-v\left(r_{e}\right)=c(e)\left(u\left(h_{e}\right)-u\left(t_{e}\right)\right) .
$$

The pair $f=(u: V \rightarrow \mathbb{R}, v: F \rightarrow \mathbb{R})$ of two conjugate functions is called a discrete analytic function. We write $\operatorname{Re} f:=u$ and $\operatorname{Im} f:=v$. If both $u$ and $v$ are constant functions, not necessarily equal to each other, we write $f=$ const. A direct checking shows that on simply connected surfaces discrete harmonic functions are precisely real parts of discrete analytic functions. Note that for non-zero weights $c(e) \neq 0$ we define the (discrete) energy of a function $v: F \rightarrow \mathbb{R}$ by

$$
E_{T}(v):=\sum_{e=[x, y] \in E} \frac{1}{c([x, y])}(v(y)-v(x))^{2} .
$$

We will consider multi-valued functions on the vertices and the faces of the triangulation $T$. Informally, a multi-valued function changes its values after performing some nontrivial loop on the surface.

Recall that the Riemann surface $\mathscr{R}$ is a branched covering of $\hat{\mathbb{C}}$ with genus $g \geqslant 1$. Denote by $p: \widetilde{\mathscr{R}} \rightarrow \mathscr{R}$ the universal covering of $\mathscr{R}$ and by $p: \widetilde{T} \rightarrow T$ 
the induced universal covering of $T$. Fix a base point $z_{0} \in \widetilde{\mathscr{R}}$ and closed paths $\alpha_{1}, \ldots, \alpha_{g}, \beta, \ldots, \beta_{g}:[0,1] \rightarrow \mathscr{R}$ forming a standard basis of the fundamental group $\pi_{1}\left(\mathscr{R}, p\left(z_{0}\right)\right)$ such that $\alpha_{1} \beta_{1} \alpha_{1}^{-1} \beta_{1}^{-1} \cdots \alpha_{g} \beta_{g} \alpha_{g}^{-1} \beta_{g}^{-1}$ is null-homotopic. An example for an algorithm to compute this basis on a computer can be found in [24, 39]. Each closed path $\gamma:[0,1] \rightarrow \mathscr{R}$ with $\gamma(0)=\gamma(1)=p\left(z_{0}\right)$ determines the deck transformation $d_{\gamma}: \widetilde{\mathscr{R}} \rightarrow \widetilde{\mathscr{R}}$, that is, the homeomorphism such that $p \circ d_{\gamma}=p$ and $d_{\gamma}\left(z_{0}\right)=\tilde{\gamma}(1)$, where $\tilde{\gamma}: \mathscr{R} \rightarrow \widetilde{\mathscr{R}}$ is the lift of $\gamma:[0,1] \rightarrow S$ such that $\tilde{\gamma}(0)=z_{0}$. The induced deck transformation of $\widetilde{T}$ is also denoted by $d_{\gamma}: \widetilde{T} \rightarrow \widetilde{T}$.

A multi-valued function with periods $A_{1}, \ldots, A_{g}, B_{1}, \ldots, B_{g} \in \mathbb{C}$ is a pair of functions $f=(\underset{\sim}{\operatorname{Re} f}: \widetilde{V} \rightarrow \mathbb{R}, \operatorname{Im} f: \widetilde{F} \rightarrow \mathbb{R})$ such that for each $k=1, \ldots, g$ an each $z \in \widetilde{V}, w \in \widetilde{F}$ we have

$$
\begin{aligned}
& \operatorname{Re} f\left(d_{\alpha_{k}}(z)\right)-\operatorname{Re} f(z)=\operatorname{Re}\left(A_{k}\right), \quad \operatorname{Re} f\left(d_{\beta_{k}}(z)\right)-\operatorname{Re} f(z)=\operatorname{Re}\left(B_{k}\right), \\
& \operatorname{Im} f\left(d_{\alpha_{k}}(z)\right)-\operatorname{Im} f(z)=\operatorname{Im}\left(A_{k}\right), \quad \operatorname{Im} f\left(d_{\beta_{k}}(z)\right)-\operatorname{Im} f(z)=\operatorname{Im}\left(B_{k}\right) .
\end{aligned}
$$

The numbers $A_{1}, \ldots, A_{g}$ and $B_{1}, \ldots, B_{g}$ are called the $A$-periods and the $B$-periods of the multi-valued function $f$, respectively. Analogously, we can also define multivalued functions $u: \widetilde{V} \rightarrow \mathbb{R}, v: \widetilde{F} \rightarrow \mathbb{R}$ or $u: \widetilde{R} \rightarrow \mathbb{R}$. Note in particular, that for each multi-valued function $u: \widetilde{V} \rightarrow \mathbb{R}$ and every edge $[x, y] \in E$ the difference $u(x)-u(y)$ is well defined. The (discrete) energy of the multi-valued function is

$$
E_{T}(u)=\sum_{[x, y] \in E} c([x, y])(u(x)-u(y))^{2} .
$$

Similarly, for each multi-valued function $u: \widetilde{\mathscr{R}} \rightarrow \mathbb{R}$, which is smooth on every face of $\widetilde{F}$, at each point inside a face $\Delta \in F$ the gradient $\nabla u$ is well defined. The (Dirichlet) energy of the multi-valued function is

$$
E(u)=\sum_{\Delta_{f} \in F} \int_{\Delta_{f}}|\nabla u|^{2} .
$$

A multi-valued discrete analytic function is called a discrete holomorphic integral or discrete Abelian integral of the first kind.

Theorem 1 [7, Theorem 2.3] For any numbers $A_{1}, \ldots, A_{g} \in \mathbb{C}$ there exists a discrete holomorphic integral with A-periods $A_{1}, \ldots, A_{g}$. It is unique up to a constant.

For each $l=1, \ldots, g$ denote by $\phi_{T}^{l}=\left(\operatorname{Re} \phi_{T}^{l}: \widetilde{V} \rightarrow \mathbb{R}, \operatorname{Im} \phi_{T}^{l}: \widetilde{F} \rightarrow \mathbb{R}\right)$ the unique (up to constant) discrete holomorphic integral with $A$-periods given by $A_{k}=$ $\delta_{k l}$, where $k=1, \ldots, g$. The $g \times g$-matrix $\Pi_{T}$ whose $l$-th column is formed by the $B$-periods of $\phi_{T}^{l}$, where $l=1, \ldots, g$, is called the period matrix of the triangulation $T$.

\subsection{Convergence of Energy and Discrete Period Matrices}

So far, we have defined our notions like discrete energy for a rather general class of triangulations. In view of our convergence results, we now make some additional assumptions. In order to measure distances and other metric properties, we always consider the images of the triangles in $\mathbb{C}$ under the charts $\operatorname{Pr}_{\mathscr{R}}$ and $\operatorname{Pr}_{\mathscr{R}}^{\infty}$. By our 
assumptions above, these are Euclidean triangles if they are contained in $B_{\rho}(0)$ after applying $\operatorname{Pr}_{\mathscr{R}}$ and in $\bar{B}_{1 / \rho}(0)$ after applying $\operatorname{Pr}_{\mathscr{R}}^{\infty}$. Therefore, we can use the standard metric in $\mathbb{C}$. We also apply this metric for boundary triangles in $F_{\rho}$ after applying $\operatorname{Pr}_{\mathscr{R}}$. Alternatively, we could work on $\hat{\mathbb{C}}$ with the chordal metric.

First we determine the maximal distance between two vertices in a triangle which lies inside $B_{\rho}(0)$ or $\bar{B}_{1 / \rho}(0)$ or on the boundary $F_{\rho}$ resp. The maximum is called maximal edge length and denoted by $h=h(T)$.

Furthermore, we suppose that near all branch points of $\mathscr{R}$ the edge lengths are adapted to the singularity which then guarantees an approximation error of order $h$. In particular, for every branch point $v_{O} \in V$ with $f\left(v_{O}\right) \neq \infty$, choose a radius $r_{O}$ such that the disks of these radii are disjoint for different points $O=\operatorname{Pr}_{\mathscr{R}}\left(v_{O}\right) \in \mathbb{C}$. Furthermore, we assume that all these disks are all contained in $B_{\rho}(0)$. Let $\mathscr{C}_{O}^{\mathscr{R}}$ be the neighborhood of $v_{O}$ in $\mathscr{R}$ which projects onto this disc $B_{r_{O}}(O)=\operatorname{Pr}_{\mathscr{R}}\left(\mathscr{C}_{O}^{\mathscr{R}}\right)$. If $O=\infty$, we first apply the the chart and $\operatorname{Pr}_{\mathscr{R}}^{\infty}$ assume that $r_{O}=1 /(2 \rho)$. For all branch points we already have a natural complex structure and charts. In particular if $O \neq \infty$, we consider the chart $g_{O}(z)=(z-O)^{\gamma_{O}}$, so $g_{O} \circ \operatorname{Pr}_{\mathscr{R}}$ maps $\mathscr{C}_{O}^{\mathscr{R}}$ onto a neighborhood of the origin in $\mathbb{C}$. If $O=\infty$, consider $g_{O}(z)=1 / z^{\gamma O}$ instead. We can also introduce "polar coordinates" $(r, \phi)$ on $\mathscr{C}_{O}^{\mathscr{R}}$ with the origin at the vertex $O$. We map all vertices of $T$ in $\mathscr{C}_{O}^{\mathscr{R}}$ to a neighborhood of the origin in $\mathbb{C}$ by the chart $g_{O}(r, \phi):=r^{\gamma_{O}} \mathrm{e}^{i \gamma_{O} \phi}$.

In any case, consider all triangles in $\mathscr{C}_{O}^{\mathscr{R}}$ which are incident to $v_{O}$. The aperture of $O$ is the sum of all the face angles at $O$ of the projection of these triangles. Denote by $\gamma_{O}$ the value $2 \pi$ divided by the aperture. Note that for branch points we have $\gamma_{O} \in\{1 / n: n=2,3,4, \ldots\}$, so $\gamma_{O} \leqslant 1 / 2$.

We demand that the triangles in the neighborhood $\mathscr{C}_{O}$ of $O$ have an adapted size: as an additional condition, we demand that

- the images under the chart $g_{O}$ of any two incident vertices in $\mathscr{C}_{O}$ have maximum distance $h$.

In particular, consider any triangle $\Delta$ in $\operatorname{Pr}_{\mathscr{R}}\left(\mathscr{C}_{O}^{\mathscr{R}}\right)\left(\right.$ or $\operatorname{Pr}_{\mathscr{R}}^{\infty}\left(\mathscr{C}_{O}^{\mathscr{R}}\right)$ if $\left.O=\infty\right)$ whose vertex $z$ nearest to $O$ satisfies $|O z| \geqslant h_{O}=h^{1 /\left(2 \gamma_{O}\right)}$, where $|O z|$ denotes the distance of $z$ to $O$ in the chart in $\mathbb{C}$. Then we deduce from our assumption that the maximal edge length in $\Delta$ is smaller than $h \cdot|O z|^{1-\gamma_{O}}$.

In Section 5 we explain how our ideas can be used for polyhedral surfaces with more general conical singularities with $0<\gamma_{O} \leqslant 1 / 2$.

We will always assume that the maximal edge length $h$ is strictly smaller than $\max \left\{\rho / 4, r_{O} / 4,1\right\}$.

A triangulation $T$ which satisfies these additional properties for all its branch points will be called adapted triangulation.

Theorem 2 (Energy convergence) For each $\delta>0$ and each smooth multi-valued harmonic function $u: \widetilde{\mathscr{R}} \rightarrow \mathbb{R}$ there are two constants Const $_{u, \delta, \mathscr{R}, \rho}$, const $_{u, \delta, \mathscr{R}, \rho}>$ 0 such that for any adapted triangulation $T$ of $\mathscr{R}$ with maximal edge length $h<$ const $_{u, \delta, \mathscr{R}, \rho}$ and minimal face angle $>\delta$ we have

$$
\left|E_{T}\left(\left.u\right|_{\tilde{V}}\right)-E(u)\right| \leqslant \text { Const }_{u, \delta, \mathscr{R}, \rho} \cdot h .
$$


The assumption on the minimal face angle in the theorem cannot be dropped, see [7, Example 4.14].

Based on energy estimates from this theorem, we deduce convergence of discrete period matrices. To this end, recall that $\mathscr{R}$ is a Riemann surface which is a branched covering of $\hat{\mathbb{C}}$. Therefore, a basis of holomorphic integrals $\phi_{\mathscr{R}}^{l}: \widetilde{\mathscr{R}} \rightarrow \mathbb{C}$ and the period matrix $\Pi_{\mathscr{R}}$ of $\mathscr{R}$ are defined analogously to the discrete case above.

Theorem 3 (Convergence of discrete period matrices) For each $\delta>0$ there are two constants Const $_{\delta, \mathscr{R}, \rho}$, const $\delta, \mathscr{R}, \rho>0$ such that for any adapted triangulation $T$ of $\mathscr{R}$ with maximal edge length $h<\operatorname{const}_{u, \delta, \mathscr{R}, \rho}$ and minimal face angle $>\delta$ we have

$$
\left\|\Pi_{T}-\Pi_{\mathscr{R}}\right\| \leqslant \text { Const }_{\delta, \mathscr{R}, \rho} \cdot h .
$$

Both theorems are proved in Section 3.

\subsection{Convergence of Discrete Holomorphic Integrals}

For the next theorem, we need some additional notions similarly as in [7]. The discrete holomorphic integral $\phi_{T}^{l}=\left(\operatorname{Re} \phi_{T}^{l}: \widetilde{V} \rightarrow \mathbb{R}, \operatorname{Im} \phi_{T}^{l}: \widetilde{F} \rightarrow \mathbb{R}\right)$ is normalized at a vertex $z \in \widetilde{T}$ and a face $w \in \widetilde{F}$, if $\operatorname{Re} \phi_{T}^{l}(z)=0=\operatorname{Im} \phi_{T}^{l}(w)$. Similarly, we call a holomorphic integral $\phi_{\mathscr{R}}^{l}: \widetilde{\mathscr{R}} \rightarrow \mathbb{C}$ normalized at a point $z \in \widetilde{\mathscr{R}}$ if $\phi_{\mathscr{R}}^{l}(z)=0$.

Recall that a triangulation $T$ is Delaunay, if for every edge $e \in E$ we have $\alpha_{e}+$ $\beta_{e} \leqslant \pi$.

Let $\left\{T_{n}\right\}$ be a sequence of adapted triangulations of the surface $\mathscr{R}$ with maximal edge length $h<\max \left\{\rho / 4, r_{0} / 4,1\right\}$. Such a sequence of adapted triangulations is called non-degenerate uniform, if there is a constant Const, not depending on $n$, such that for each member of the sequence:

(A) the angles of each triangle are greater than $1 /$ Const.

(D) for each edge the sum of opposite angles in the two triangles containing the edge is less than $\pi-1 /$ Const. (In particular, the triangulation is Delaunay within $B_{\rho}(0)$ and within $\bar{B}_{1 / \rho}(0)$.)

(U) the number of vertices in an arbitrary intrinsic disc about $z$ of radius equal to the maximal edge length is smaller than Const if $z$ is not contained in any of the neighborhoods $\mathscr{C}_{O}$ of a singularity $O$. Within such a neighborhood $\mathscr{C}_{O}$, first map the vertices to a disc about the origin by the map $\zeta \mapsto(\zeta-O)^{\gamma O}$ if $O \neq \infty$ (or $\zeta \mapsto 1 / \zeta^{\gamma O}$ if $O=\infty$ ). Then we require that after this mapping in each disc of radius equal to the maximal edge length the number of image points of vertices is smaller than Const.

A sequence of functions $f_{n}=\left(\operatorname{Re} f_{n}: \widetilde{V}_{n} \rightarrow \mathbb{R}, \operatorname{Im} f_{n}: \widetilde{F}_{n} \rightarrow \mathbb{R}\right)$ converges to a function $f: \widetilde{\mathscr{R}} \rightarrow \mathbb{C}$ uniformly on compact subsets if for every compact set $K \subset \widetilde{\mathscr{R}}$ we have

$$
\begin{aligned}
& \max _{z \in K \cap \widetilde{V}_{n}}\left|\operatorname{Re} f_{n}(z)-\operatorname{Re} f(z)\right| \rightarrow 0 \quad \text { and } \\
& \max _{\Delta[x, y, z] \in F, \Delta[x, y, z] \cap K \neq \emptyset}\left|\operatorname{Im} f_{n}(\Delta[x, y, z])-\operatorname{Im} f(z)\right| \rightarrow 0 \quad \text { as } n \rightarrow \infty .
\end{aligned}
$$


Theorem 4 (Convergence of discrete holomorphic integrals) Let $\left\{T_{n}\right\}$ be a sequence of non-degenerate uniform adapted triangulations of $\mathscr{R}$ with maximal edge length $h_{n} \rightarrow \underset{\mathscr{R}}{0}$ as $n \rightarrow \infty$. Let $z_{n} \in \widetilde{V}_{n}$ be a sequence of vertices converging to a point $z_{0} \in \widetilde{\mathscr{R}}$. Let $\Delta_{n} \in \widetilde{F}_{n}$ be a sequence of faces with its vertices converging to $z_{0}$. Then for each $1 \leqslant l \leqslant g$ the discrete holomorphic integrals $\phi_{T}^{l}=\left(\operatorname{Re} \phi_{T}^{l}: \widetilde{V} \rightarrow\right.$ $\left.\mathbb{R}, \operatorname{Im} \phi_{T}^{l}: \widetilde{F} \rightarrow \mathbb{R}\right)$ normalized at $z_{n}$ and $\Delta_{n}$ converge uniformly on each compact set to the holomorphic integral $\phi_{\mathscr{R}}^{l}: \widetilde{\mathscr{R}} \rightarrow \mathbb{C}$ normalized at $z_{0}$.

This theorem is proved in Section 4.

\section{Proof of Convergence of Energy and Period Matrices}

In this section, we prove convergence of the discrete energy to the corresponding Dirichlet energy and convergence of discrete period matrices to their continuous counterparts. The main ideas of the proof follow [7, Section 4], but we improve the estimates near branch points (which can be considered as special conical singularities) by using the additional properties of the adapted triangulations.

In the following, all triangles being considered are in $\mathbb{C}$ after application of $\operatorname{Pr}_{\mathscr{R}}$ or $\operatorname{Pr}_{\mathscr{R}}^{\infty}$ resp.

We denote by Const ${ }_{a, b, c}$ a positive constant which only depends on the parameters $a, b, c$. The symbol Const may denote distinct constants at different places of the text, for example in 2 . Const $\leqslant$ Const. Furthermore, we set $\left\|D^{k} u(z)\right\|:=$ $\max _{0 \leqslant j \leqslant k}\left|\frac{\partial^{k} u}{\partial^{j} x \partial^{k-j} y}(z)\right|$.

\subsection{Energy Estimates in a Triangle}

First we consider only one triangle $\Delta$ of the triangulation $T$. Let $u: \Delta \rightarrow \mathbb{R}$ be a smooth function which smoothly extends to a neighborhood of $\Delta$. Let $I_{T} u: \Delta \rightarrow$ $\mathbb{R}$ be the corresponding interpolation function defined in Section 2.1. Then we set $E_{\Delta}(u)=\int_{\Delta}|\nabla u|^{2} d x d y$ and $E_{T_{\Delta}}(u)=\int_{\Delta}\left|\nabla I_{T} u\right|^{2} d x d y$. Denote by $\delta$ the minimal angle of the triangle $\Delta$.

Lemma 2 (Energy approximation on a triangle) (i) If the triangle $\Delta$ is contained in $B_{\rho}(0)$ under the chart $\operatorname{Pr}_{\mathscr{R}}$ or in the closed disc $\bar{B}_{1 / \rho}(0)$ under the chart $\operatorname{Pr}_{\mathscr{R}}^{\infty}$, denote by $l_{\max }$ the maximum edge length of $\Delta$. Then

$$
\begin{aligned}
\left|E_{T_{\Delta}}(u)-E_{\Delta}(u)\right| \leqslant & \text { Const }_{\delta} \cdot\left(\max _{w \in \Delta}\left\|D^{1} u(w)\right\|+l_{\max } \cdot \max _{w \in \Delta}\left\|D^{2} u(w)\right\|\right) \\
& \cdot l_{\max } \cdot \max _{w \in \Delta}\left\|D^{2} u(w)\right\| \cdot \operatorname{Area}(\Delta) .
\end{aligned}
$$

(ii) If the triangle $\Delta$ is a boundary triangle in $F_{\rho}$ then

$$
\left|E_{T_{\Delta}}(u)-E_{\Delta}(u)\right| \leqslant \operatorname{Const}_{\delta, \rho} \cdot \max _{w \in \Delta}\left\|D^{1} u(w)\right\|^{2} \cdot \operatorname{Area}(\Delta)
$$


Proof (i) For triangles contained in $B_{\rho}(0)$ under the chart $\operatorname{Pr}_{\mathscr{R}}$ or in the closed disc $\bar{B}_{1 / \rho}(0)$ under the chart $\operatorname{Pr}_{\mathscr{R}}^{\infty}$ this is Lemma 4.4 in [7].

(ii) For boundary triangles we estimate the discrete and the smooth energy separately. For the smooth energy, we have $E_{\Delta}(u) \leqslant$ Const $\cdot \max _{w \in \Delta}\left\|D^{1} u(w)\right\|^{2}$. $\operatorname{Area}(\Delta)$. For the discrete energy, an estimate of $E_{T_{\Delta}}(u)$ is contained in Appendix A.2, see in particular (16).

\subsection{Energy Estimates Near a Branch Point}

Let $v_{O} \in \mathscr{R}$ be a branch point of $\mathscr{R}$ with $\gamma_{O} \leqslant 1 / 2$. In this subsection we only consider those triangles of the adapted triangulation $T$ which are completely contained in the neighborhood $\mathscr{C}_{O}^{\mathscr{R}}$. Denote by $T_{O}$ the connected component of these triangles which contains $v_{O}$. For the estimate of the difference of energies for these triangles we consider in particular $E_{T_{O}}(u)=\sum_{\Delta \in F_{O}} E_{T_{\Delta}}(u)$ and $E_{S_{O}}(u)=\sum_{\Delta \in F_{O}} E_{\Delta}(u)$, where $F_{O}$ denotes the set of triangles in $T_{O}$ and $S_{O}$ is the neighborhood of $v_{O}$ covered by these triangles.

As the partial derivatives of $u$ (considered in a chart) are not necessarily bounded near the vertex $O$, we consider triangles in a 'very small' neighborhood of $O$ separately. Let $S_{O, h_{O}}$ be the union of faces of $T_{O}$ whose images under $\operatorname{Pr}_{\mathscr{R}}$ intersect the disc of radius $h_{O}:=h^{1 /\left(2 \gamma_{O}\right)}$ about $O$ and let $T_{O, h_{O}}$ be the restriction of $T_{O}$ to $S_{O, h_{O}}$. Denote by $F_{O, h_{O}}$ the set of faces of $T_{O, h_{O}}$. Note that we use polar coordinates $(\rho, \phi)$ as introduced in Section 2.3 as a chart for $\mathscr{C}_{O}^{\mathscr{R}}$.

Lemma 3 (Derivative Estimation Lemma, [7, Lemma 4.5]) For each $w=(\rho, \phi) \in$ $\mathscr{C}_{O}^{\mathscr{R}}$ such that $w \neq O$ we have

$\left\|\left.D^{1} u(z)\right|_{w}\right\| \leqslant$ const $_{u, r_{O}, \gamma_{O}} \cdot \rho^{\gamma_{O}-1} \quad$ and $\quad\left\|\left.D^{2} u(z)\right|_{w}\right\| \leqslant \operatorname{const}_{u, r_{O}, \gamma_{O}} \cdot \rho^{\gamma_{O}-2}$.

Lemma 4 [7, Lemma 4.12] For every $\Delta \in T_{O, h_{O}}$ we have $E_{T_{\Delta}}(u) \leqslant$ Const $_{\delta, \gamma_{O}, r_{O}, u}$. $\int_{\Delta} \rho^{2 \gamma_{0}-1} d \rho d \phi$.

Lemma 5 For every $\Delta \in T_{O} \backslash T_{O, h_{O}}$ we have $\left|E_{T_{\Delta}}(u)-E_{\Delta}(u)\right| \leqslant$ Const $_{\delta, \gamma_{O}, r_{O}, u}$. $h \cdot \int_{\Delta} \rho^{\gamma_{O}-1} d \rho d \phi$.

Proof Let $z \in \Delta$ be the vertex closest to $O$. As $\Delta \in F_{O} \backslash F_{O, h_{O}}$ we have for each point $(\rho, \phi)$ in $\Delta$ that

$$
h^{1 / \gamma_{O}} \leqslant h^{1 /\left(2 \gamma_{O}\right)} \leqslant|O z| \leqslant \rho \leqslant|O z|+h|O z|^{1-\gamma_{O}} \leqslant 2|O z| .
$$

This also implies $h \leqslant|O z|^{\gamma_{O}}$. Furthermore, on $F_{O} \backslash F_{O, h_{O}}$ we can use Lemma 3 together with our assumption that the edge lengths in $\Delta$ are smaller than $h|O z|^{1-\gamma_{O}}$. If we also apply Lemma 2 and the above estimates on $\rho$, we obtain

$$
\left|E_{T_{\Delta}}(u)-E_{\Delta}(u)\right| \leqslant \text { Const }_{\delta, \gamma_{O}, r_{O}, u}(|O z|^{\gamma_{O}-1}+\underbrace{h|O z|^{1-\gamma_{O}} \cdot|O z|^{\gamma_{O}-2}}_{\leqslant|O z|^{\gamma_{O}-1}})
$$




$$
\begin{array}{r}
\cdot h|O z|^{1-\gamma_{O}} \cdot|O z|^{\gamma_{O}-2} \cdot \underbrace{\operatorname{Area}(\Delta)}_{=\int_{\Delta} \rho d \rho d \phi} \\
\leqslant \text { Const }_{\delta, \gamma_{O}, r_{O}, u} \cdot h \cdot \int_{\Delta} \rho^{\gamma_{O}-1} d \rho d \phi .
\end{array}
$$

Lemma 6 We have $\left|E_{T_{O} \backslash T_{O, h_{O}}}(u)-E_{S_{O} \backslash S_{O, h_{O}}}(u)\right| \leqslant$ Const $_{\delta, \gamma_{O}, r_{O}, u} \cdot h$.

Proof We use Lemma 5, sum the inequalities and estimate the integral.

$$
\begin{aligned}
\left|E_{T_{O} \backslash T_{O, h_{O}}}(u)-E_{S_{O} \backslash S_{O, h_{O}}}(u)\right| & \leqslant \sum_{\Delta \in F_{O} \backslash F_{O, h_{O}}}\left|E_{T_{\Delta}}(u)-E_{\Delta}(u)\right| \\
& \leqslant \operatorname{Const}_{\delta, \gamma_{O}, r_{O}, u} \cdot h \cdot \int_{S_{O} \backslash S_{O, h_{O}}} \rho^{\gamma_{O}-1} d \rho \\
& \leqslant \text { Const }_{\delta, \gamma_{O}, r_{O}, u} \cdot h
\end{aligned}
$$

Now we estimate the energies on $S_{O, h_{O}}$ and $F_{O, h_{O}}$ separately.

Lemma 7 We have $E_{S_{O, h_{O}}}(u) \leqslant$ Const $_{\gamma_{O}, u} \cdot h$.

Proof Using Lemma 3 and our definition of $S_{O, h_{O}}$ we obtain

$$
\begin{aligned}
E_{S_{O, h_{O}}}(u)= & \sum_{\Delta \in F_{O, h_{O}}} \int_{\Delta}|\nabla u|^{2} d x d y \leqslant \text { Const }_{\gamma_{O}, u} \int_{\phi=0}^{2 \pi / \gamma_{O}} \int_{\rho=0}^{2^{1 /\left(2 \gamma_{O}\right)} h^{1 /\left(2 \gamma_{O}\right)}} \\
& \rho^{2 \gamma_{O}-1} d \rho d \phi \leqslant \text { Const }_{\gamma_{O}, u} \cdot h .
\end{aligned}
$$

Lemma 8 We have $E_{T_{O, h_{O}}}(u) \leqslant$ Const $_{\delta, \gamma_{O}, u} \cdot h$.

Proof We deduce from Lemma 4 similarly as in the previous lemma that

$$
\begin{aligned}
E_{F_{O, h_{O}}}(u)= & \sum_{\Delta \in F_{O, h_{O}}} \int_{\Delta}\left|\nabla I_{T} u\right|^{2} d x d y \leqslant \text { Const }_{\delta, \gamma_{O}, u} \int_{\phi=0}^{2 \pi / \gamma_{O}} \int_{\rho=0}^{2^{1 /\left(2 \gamma_{O}\right)} h^{1 /\left(2 \gamma_{O}\right)}} \\
& \rho^{2 \gamma_{O}-1} d \rho d \phi \leqslant \text { Const }_{\delta, \gamma_{O}, u} \cdot h .
\end{aligned}
$$

\subsection{Convergence of Energies}

Let $G_{T}=F \backslash\left\{F_{\rho} \cup \bigcup_{O \text { branch point }} F_{O}\right\}$ be the set of triangles which are neither contained in the neighborhood of any branch point nor are boundary triangles. Denote by $G$ the subset of $\mathscr{R}$ covered by the triangles in $G_{T}$.

Lemma 9 We have $\left|E_{G_{T}}(u)-E_{G}(u)\right| \leqslant$ Const $_{\delta, u, \mathscr{R}, \rho} \cdot h$. 
Proof By applying the charts $\operatorname{Pr}_{\mathscr{R}}$ and $\operatorname{Pr}_{\mathscr{R}}^{\infty}$ respectively, the triangles of $G_{T}$ are mapped into the intrinsic disc $B_{\rho}(0)$ of radius $\rho$ and intrinsic disc $\bar{B}_{1 / \rho}(0)$ of radius $1 / \rho$ about the origin respectively.

Our assumption on the maximal edge length, the definition of the discrete energy, the compactness of $\mathscr{R}$, and the estimates in Lemma 2 imply that

$$
\begin{aligned}
\left|E_{G_{T}}(u)-E_{G}(u)\right| & \leqslant \sum_{\Delta \in G_{T}}\left|E_{T_{\Delta}}(u)-E_{\Delta}(u)\right| \\
& \leqslant \sum_{\Delta \in G_{T}} \operatorname{Const}_{\delta, u, \rho} \cdot(1+h) \cdot h \cdot \operatorname{Area}(\Delta) \\
& \leqslant \sum_{\Delta \in G_{T}} \operatorname{Const}_{\delta, u, \rho} \cdot h \cdot \int_{\Delta} \rho d \rho d \phi \leqslant \text { Const }_{\delta, u, \rho} \cdot h \cdot \int_{G} \rho d \rho \\
& \leqslant \text { Const }_{\delta, u, \mathscr{R}, \rho} \cdot h .
\end{aligned}
$$

Lemma 10 We have $E_{S_{\rho}}(u) \leqslant$ Const $_{u, \rho} \cdot h$, where $S_{\rho}$ denotes the subset of $\mathscr{R}$ which is covered by triangles of $F_{\rho}$.

Proof This estimate is due to the fact that the derivative of $u$ is bounded away from the branch points. Furthermore, the area of the ring $\{\rho-h \leqslant|z| \leqslant \rho+h\}$ is bounded by $4 \pi \rho h$ and the degree of the branched covering is fixed. Therefore,

$$
E_{S_{\rho}}(u)=\int_{S_{\rho}}|\nabla u|^{2} d x d y \leqslant \text { Const }_{u, \rho, \mathscr{R}} \cdot h .
$$

Lemma 11 We have $E_{F_{\rho}}(u):=\sum_{\Delta \in F_{\rho}} E_{T_{\Delta}}(u) \leqslant$ Const $_{u, \delta, \rho, \mathscr{R}} \cdot h$.

The proof of this lemma is given in Appendix A.2.

Proof of Theorem 2 Summing up the estimates obtained in Lemmas 6-11 we get the desired result:

$$
\begin{aligned}
\left|E_{T}\left(\left.u\right|_{\tilde{V}}\right)-E(u)\right| \leqslant & \left|E_{G_{T}}(u)-E_{G}(u)\right|+E_{F_{\rho}}(u)+\sum_{O \text { branch point of } \mathscr{R}}\left(\mid E_{T_{O} \backslash T_{O, h_{O}}}(u)\right. \\
& \left.-E_{S_{O} \backslash S_{O, h_{O}}}(u) \mid+E_{S_{O, h_{O}}}(u)+E_{T_{O, h_{O}}}(u)\right) \\
\leqslant & \text { Const }_{\delta, u, \mathscr{R}, \rho} \cdot h .
\end{aligned}
$$

\subsection{Convergence of Discrete Period Matrices}

For our convergence proof we start with some further useful theorems and definitions.

Lemma 12 (Variational principle [7, Lemma 3.6]) A multi-valued discrete harmonic function has minimal energy among all multi-valued functions with the same periods.

Theorem 5 [7, Theorem 3.9] For each $P=\left(A_{1}, \ldots, A_{g}, B_{1}, \ldots, B_{g}\right) \in \mathbb{R}^{2 g}$ there exists a unique (up to a constant) discrete holomorphic integral $\phi_{T, P}=\left(\operatorname{Re} \phi_{T, P}\right.$ : 
$\left.\widetilde{V} \rightarrow \mathbb{R}, \operatorname{Im} \phi_{T, P}: \widetilde{F} \rightarrow \mathbb{R}\right)$ whose periods have real parts $A_{1}, \ldots, A_{g}, B_{1}, \ldots, B_{g}$, respectively.

Denote $u_{T, P}=\operatorname{Re} \phi_{T, P}$, where $\phi_{T, P}$ is the discrete holomorphic integral defined in Theorem 5 for each vector $P \in \mathbb{R}^{2 g}$. Analogously, let $\phi_{\mathscr{R}, P}: \widetilde{\mathscr{R}} \rightarrow \mathbb{C}$ be a holomorphic integral whose periods have real parts $A_{1}, \ldots, A_{g}, B_{1}, \ldots, B_{g}$, respectively. Denote $u_{\mathscr{R}, P}=\operatorname{Re} \phi_{\mathscr{R}, P}$.

Lemma 13 For every $\delta>0$ and every vector $P \in \mathbb{R}^{2 g}$ there are constants Const $_{P, \delta, \mathscr{R}, \rho}$, const $P, \delta, \mathscr{R}, \rho>0$ such that for any adapted triangulation $T$ of $\mathscr{R}$ with maximal edge length $h<\operatorname{const}_{P, \delta, \mathscr{R}, \rho}$ and minimal face angle $\delta>0$ we have

$$
\left|E_{T}\left(u_{T, P}\right)-E\left(u_{\mathscr{R}, P}\right)\right| \leqslant \text { Const }_{P, \delta, \mathscr{R}, \rho} \cdot h .
$$

Proof From the interpolation Lemma 1 we know that $E_{T}\left(u_{T, P}\right)=E\left(I_{T} u_{T, P}\right)$ and the interpolation function $I_{T} u_{T, P}$ is continuous and piecewise smooth. Using Lemma 12 and its smooth counterpart (Dirichlet's principle) we deduce from Theorem 2 that

$$
\begin{aligned}
0 & \leqslant E\left(I_{T} u_{T, P}\right)-E\left(u_{\mathscr{R}, P}\right)=E_{T}\left(u_{T, P}\right)-E\left(u_{\mathscr{R}, P}\right) \leqslant E_{T}\left(\left.u_{\mathscr{R}, P}\right|_{\widetilde{V}}\right)-E\left(u_{\mathscr{R}, P}\right) \\
& \leqslant \operatorname{Const}_{P, \delta, \mathscr{R}, \rho} \cdot h .
\end{aligned}
$$

For each $l=1, \ldots, g$ denote by $\phi_{T^{*}}^{l}=\left(\operatorname{Re} \phi_{T^{*}}^{l}: \widetilde{V} \rightarrow \mathbb{R}, \operatorname{Im} \phi_{T^{*}}^{l}: \widetilde{F} \rightarrow \mathbb{R}\right)$ the unique (up to constant) discrete holomorphic integral with $A$-periods given by $A_{k}=i \delta_{k l}$, where $k=1, \ldots, g$. The $g \times g$-matrix $\Pi_{T^{*}}$ whose $l$-th column is formed by the $B$-periods of $\phi_{T^{*}}^{l}$ divided by $i$, where $l=1, \ldots, g$, is called the dual period matrix of the triangulation $T$.

The following theorem connects the period matrices to the energies.

\section{Lemma 14 [7, Lemmas $3.14 \& 3.15]$}

(i) The energy $E_{T}\left(u_{T, P}\right)$ is a quadratic form in the vector $P \in \mathbb{R}^{2 g}$ with the block matrix

$$
E_{T}:=\left(\begin{array}{cc}
\operatorname{Re} \Pi_{T^{*}}\left(\operatorname{Im} \Pi_{T^{*}}\right)^{-1} \operatorname{Re} \Pi_{T}+\operatorname{Im} \Pi_{T} & -\left(\operatorname{Im} \Pi_{T^{*}}\right)^{-1} \operatorname{Re} \Pi_{T} \\
-\operatorname{Re} \Pi_{T^{*}}\left(\operatorname{Im} \Pi_{T^{*}}\right)^{-1} & \left(\operatorname{Im} \Pi_{T^{*}}\right)^{-1}
\end{array}\right) .
$$

(ii) The energy $E\left(u_{\mathscr{R}, P}\right)$ is a quadratic form in the vector $P \in \mathbb{R}^{2 g}$ with the block matrix

$$
E_{\mathscr{R}}:=\left(\begin{array}{cc}
\operatorname{Re} \Pi_{\mathscr{R}}\left(\operatorname{Im} \Pi_{\mathscr{R}}\right)^{-1} \operatorname{Re} \Pi_{\mathscr{R}}+\operatorname{Im} \Pi_{\mathscr{R}}-\left(\operatorname{Im} \Pi_{\mathscr{R}}\right)^{-1} \operatorname{Re} \Pi_{\mathscr{R}} \\
-\operatorname{Re} \Pi_{\mathscr{R}}\left(\operatorname{Im} \Pi_{\mathscr{R}}\right)^{-1} & \left(\operatorname{Im} \Pi_{\mathscr{R}}\right)^{-1}
\end{array}\right) .
$$

Combining Lemmas 13 and 14, we obtain:

Corollary 1 Let $\left\{T_{n}\right\}$ be a nondegenerate uniform sequence of adapted triangulations of $\mathscr{R}$ with maximal edge length tending to zero as $n \rightarrow \infty$. Let $P_{n} \in \mathbb{R}^{2 g}$ be a sequence of $2 g$-dimensional real vectors converging to a vector $P \in \mathbb{R}^{2 g}$. Then $E_{T_{n}}\left(u_{T_{n}, P_{n}}\right) \rightarrow E\left(u_{\mathscr{R}, P}\right)$ as $n \rightarrow \infty$. 
Proof of Theorem 3. Both $E_{T_{n}}\left(u_{T_{n}, P}\right)$ and $E\left(u_{\mathscr{R}, P}\right)$ are quadratic forms in $P \in \mathbb{R}^{2 g}$ by Lemma 14 with block matrices $E_{T}$ and $E_{\mathscr{R}}$, respectively. Thus by Lemma 13 for every $\delta>0$ there are constants Const $_{\delta, \mathscr{R}, \rho}$, const $_{\delta, \mathscr{R}, \rho}>0$ such that for any adapted triangulation $T$ of $\mathscr{R}$ with maximal edge length $h<$ const $_{\delta, \mathscr{R}, \rho}$ and minimal face angle $\delta>0$ we have $\left\|E_{T}-E_{\mathscr{R}}\right\| \leqslant$ Const $_{\delta, \mathscr{R}, \rho} \cdot h$. From this inequality we deduce estimates on $\left\|\operatorname{Re} \Pi_{T}-\operatorname{Re} \Pi_{\mathscr{R}}\right\|$ and $\left\|\operatorname{Im} \Pi_{T}-\operatorname{Im} \Pi_{\mathscr{R}}\right\|$ of the same type, but with different constants which are derived in the following. These estimates complete the proof.

- As $\left\|\left(\operatorname{Im} \Pi_{T^{*}}\right)^{-1}-\left(\operatorname{Im} \Pi_{\mathscr{R}}\right)^{-1}\right\| \leqslant$ Const $_{\delta, \mathscr{R}, \rho} \cdot h$ for $h<$ const $_{\delta, \mathscr{R}, \rho}$ there exist new constants Const ${ }_{\delta, \mathscr{R}, \rho}^{\prime}>0$ and const $_{\delta, \mathscr{R}, \rho}>$ const $_{\delta, \mathscr{R}, \rho}^{\prime}>0$ such that $\left\|\operatorname{Im} \Pi_{T^{*}}\right\| \leqslant$ Const $_{\delta, \mathscr{R}, \rho}^{\prime}$ for $h<$ const $_{\delta, \mathscr{R}, \rho}^{\prime}$.

- Thus for $h<$ const $_{\delta, \mathscr{R}, \rho}^{\prime}$ we deduce

$$
\begin{aligned}
\text { Const }_{\delta, \mathscr{R}, \rho} \cdot h \geqslant & \left\|\left(\operatorname{Im} \Pi_{T^{*}}\right)^{-1} \operatorname{Re} \Pi_{T}-\left(\operatorname{Im} \Pi_{\mathscr{R}}\right)^{-1} \operatorname{Re} \Pi_{\mathscr{R}}\right\| \\
= & \|\left(\operatorname{Im} \Pi_{T^{*}}\right)^{-1}\left(\operatorname{Re} \Pi_{T}-\operatorname{Re} \Pi_{\mathscr{R}}\right) \\
& -\left(\left(\operatorname{Im} \Pi_{\mathscr{R}}\right)^{-1}-\left(\operatorname{Im} \Pi_{T^{*}}\right)^{-1}\right) \operatorname{Re} \Pi_{\mathscr{R}} \| \\
\geqslant & \left\|\left(\operatorname{Im} \Pi_{T^{*}}\right)^{-1}\right\| \cdot\left\|\operatorname{Re} \Pi_{T}-\operatorname{Re} \Pi_{\mathscr{R}}\right\| \\
& -\left\|\left(\operatorname{Im} \Pi_{T^{*}}\right)^{-1}-\left(\operatorname{Im} \Pi_{\mathscr{R}}\right)^{-1}\right\| \cdot\left\|\operatorname{Re} \Pi_{\mathscr{R}}\right\| \\
\geqslant & \left(\text { Const }_{\delta, \mathscr{R}, \rho}^{\prime}\right)^{-1} \cdot\left\|\operatorname{Re} \Pi_{T}-\operatorname{Re} \Pi_{\mathscr{R}}\right\| \\
& - \text { Const }_{\delta, \mathscr{R}, \rho} \cdot h \cdot\left\|\operatorname{Re} \Pi_{\mathscr{R}}\right\| .
\end{aligned}
$$

Therefore, $\left\|\operatorname{Re} \Pi_{T}-\operatorname{Re} \Pi_{\mathscr{R}}\right\| \leqslant$ Const $_{\delta, \mathscr{R}, \rho}^{\prime \prime} \cdot h$, where Const $_{\delta, \mathscr{R}, \rho}^{\prime \prime}=$ Const $_{\delta, \mathscr{R}, \rho}^{\prime} \cdot$ Const $_{\delta, \mathscr{R}, \rho} \cdot\left(1+\left\|\operatorname{Re} \Pi_{\mathscr{R}}\right\|\right)$.

Analogously, we see that $\left\|\operatorname{Re} \Pi_{T^{*}}-\operatorname{Re} \Pi_{\mathscr{R}}\right\| \leqslant$ Const $_{\delta, \mathscr{R}, \rho}^{\prime \prime} \cdot h$.

- By similar estimates as for the previous item, we obtain

$$
\left\|\operatorname{Re} \Pi_{T^{*}}\left(\operatorname{Im} \Pi_{T^{*}}\right)^{-1} \operatorname{Re} \Pi_{T}-\operatorname{Re} \Pi_{\mathscr{R}}\left(\operatorname{Im} \Pi_{\mathscr{R}}\right)^{-1} \operatorname{Re} \Pi_{\mathscr{R}}\right\| \leqslant \operatorname{Const}_{\delta, \mathscr{R}, \rho}^{\prime \prime \prime} \cdot h,
$$

where Const ${ }_{\delta, \mathscr{R}, \rho}^{\prime \prime \prime}=$ Const $_{\delta, \mathscr{R}, \rho}^{\prime \prime} \cdot\left\|\left(\operatorname{Im} \Pi_{\mathscr{R}}\right)^{-1}\right\|\left(1+2\left\|\operatorname{Re} \Pi_{\mathscr{R}}\right\|\right)+$ Const $_{\delta, \mathscr{R}, \rho} \cdot$ $\left(\text { Const }_{\delta, \mathscr{R}, \rho}^{\prime \prime}+\left\|\operatorname{Re} \Pi_{\mathscr{R}}\right\|\right)^{2}$.

- Finally, we deduce from

$$
\begin{aligned}
& \left\|\operatorname{Re} \Pi_{T^{*}}\left(\operatorname{Im} \Pi_{T^{*}}\right)^{-1} \operatorname{Re} \Pi_{T}+\operatorname{Im} \Pi_{T}-\operatorname{Re} \Pi_{\mathscr{R}}\left(\operatorname{Im} \Pi_{\mathscr{R}}\right)^{-1} \operatorname{Re} \Pi_{\mathscr{R}}-\operatorname{Im} \Pi_{\mathscr{R}}\right\| \\
\leqslant & \text { Const }_{\delta, \mathscr{R}, \rho} \cdot h
\end{aligned}
$$

together with the previous estimate that $\left\|\operatorname{Im} \Pi_{T}-\operatorname{Im} \Pi_{\mathscr{R}}\right\| \leqslant\left(\right.$ Const $_{\delta, \mathscr{R}, \rho}^{\prime \prime \prime}+$ Const $\left._{\delta, \mathscr{R}, \rho}\right) \cdot h$.

\section{Proof of Convergence of Discrete Holomorphic Integrals}

The strategy of the proof of Theorem 4 follows the corresponding ideas in [7, Section 5]. Due to our different setup, we need some modifications. 


\subsection{Equicontinuity}

In this section we consider triangulations $T^{\prime}$ of branched coverings with boundary. The main goal is to consider (sufficiently small) intrinsic discs about a branch points or about a regular point and derive an estimate for harmonic functions there. A function $u: V^{\prime} \rightarrow \mathbb{R}$ is discrete harmonic on $T^{\prime}$ if it satisfies (2) at every non-boundary vertex. Denote $E_{T^{\prime}}^{\prime}(u)=\sum_{e=[x, y] \in E^{\prime} \backslash \partial E^{\prime}} c([x, y])(u(y)-u(x))^{2}$, where the sum is over non-boundary edges. Let the eccentricity $e$ denote the number Const such the triangulation $T$ satisfies conditions (A), (D), (U) from Section 2.4, where (A) and (D) only hold for every non-boundary edge.

Let $T$ be a non-degenerate uniform adapted triangulation of the branched covering of $\mathscr{R}$. We assume that $T^{\prime}$ is a simply connected part of $T$. For simplicity, we directly consider the projection of all triangles into $\mathbb{C}$ by $\operatorname{Pr}_{\mathscr{R}}$ or $\operatorname{Pr}_{\mathscr{R}}^{\infty}$ resp.

Lemma 15 (Equicontinuity lemma) (i) Let $T^{\prime}$ be contained in an open disc $B_{r}(v) \subset \mathbb{C}$ where $2 r$ is smaller than the minimum distance of $v$ to any branch point, but $r \geqslant 10 \cdot h$. Denote by $h^{\prime}$ twice the maximum circumradius of the triangles of $T^{\prime}$. Let $u: V^{\prime} \rightarrow \mathbb{R}$ be a discrete harmonic function. Let $z, w \in V^{\prime}$ with Euclidean distance $|z-w| \geqslant h^{\prime}$ and such that $3|z-w|<r<\operatorname{dist}\left(z w, \partial T^{\prime}\right)$ for some $r>0$. Here dist $\left(z w, \partial T^{\prime}\right)$ denotes the distance of the straight line segment from $z$ to $w$ to the boundary of $T^{\prime}$. Then there exists a constant Const $_{e}>0$ such that

$$
|u(z)-u(w)| \leqslant \text { Const }_{e} \cdot E_{T^{\prime}}^{\prime}(u)^{1 / 2} \cdot\left(\log \frac{r}{3|z-w|}\right)^{-1 / 2} .
$$

For $|z-w|<h^{\prime}<r / 3$ the same inequality holds with $|z-w|$ replaced by $h^{\prime}$.

(ii) Let $T^{\prime}$ be contained in an open intrinsic disc $B_{r_{O}}(O) \subset \mathbb{C}$ about some branch points $O \neq \infty$. Let $u: V^{\prime} \rightarrow \mathbb{R}$ be a discrete harmonic function.

Consider the chart $g_{O}(z)=(z-O)^{\gamma o}$, which maps the triangulation $T^{\prime}$ contained in $\mathscr{C}_{O}$ to an embedded triangulation $T_{g}^{\prime}$ in a neighborhood of the origin in $\mathbb{C}$. Denote by $h^{\prime}$ twice the maximum circumradius of the triangles of $T_{g}^{\prime}$. Let $z, w \in V^{\prime}$ with Euclidean distance $\left|g_{O}(z)-g_{O}(w)\right|=$ $\left|(z-O)^{\gamma O}-(w-O)^{\gamma O}\right| \geqslant h^{\prime}$ and such that $3\left|(z-O)^{\gamma_{O}}-(w-O)^{\gamma O}\right|<$ $r<\operatorname{dist}\left(g_{O}(z) g_{O}(w), \partial T_{g}^{\prime}\right)$ for some $r>0$. Here $\operatorname{dist}\left(g_{O}(z) g_{O}(w), \partial T^{\prime}\right)$ denotes the distance of the straight line segment from $g_{O}(z)$ to $g_{O}(w)$ to the boundary of $T_{g}^{\prime}$. Then there exists a constant Const $_{e}>0$ such that

$$
|u(z)-u(w)| \leqslant \operatorname{Const}_{e} \cdot E_{T^{\prime}}^{\prime}(u)^{1 / 2} \cdot\left(\log \frac{r}{3\left|(z-O)^{\gamma O}-(w-O)^{\gamma O}\right|}\right)^{-1 / 2} .
$$

For $\left|(z-O)^{\gamma o}-(w-O)^{\gamma o}\right|<h^{\prime}<r / 3$ the same inequality holds with $\left|(z-O)^{\gamma_{O}}-(w-O)^{\gamma_{O}}\right|$ replaced by $h^{\prime}$.

(iii) If $O=\infty$ is a branch point, an analogous estimate as in case (ii) holds for all triangles in the open intrinsic disc $B_{r_{O}}(O) \subset \mathbb{C}$ after applying the chart $\operatorname{Pr}_{\mathscr{R}}^{\infty}$. 
Proof The claims are proved analogously to a similar estimate for quadrilateral lattices in the plane [37, Equicontinuity Lemma 2.4], see also [37, Section 1 and Remarks 3.4 and 4.8], using the approach of [26, Section 5.4]. For the sake of completeness, we present a proof in Appendix A.3.

In case (ii), we consider the harmonic function $u$ as defined on the image triangulation $T_{g}^{\prime}$. The proof only uses the fact that $u$ satisfies a maximum principle which still holds in our case. For the third case, we just work with the triangulation $T_{1 / z}^{\prime}$ under the projection $\operatorname{Pr}_{\mathscr{R}}^{\infty}$.

Lemma 16 Let $T$ be a triangulation of a ramified covering with boundary such that all angles are in $[\delta, \pi-\delta]$ for some $\pi / 4>\delta>0$. Then there exist constants const $_{\delta, \rho}$, Const $\delta, \rho>0$ such that for $0<h<$ const $_{\delta, \rho}$ and every function $u: V \rightarrow \mathbb{R}$ we have $E_{T^{\prime}}^{\prime}(u) \leqslant$ Const $_{\delta, \rho} \cdot E_{T}(u)$.

Proof Let $\Delta \in F^{\prime}$ be a triangle with vertices $x, y, z \in V$ such that $[x, z]$ is a boundary edge of $T^{\prime}$. Denote the angle in $\Delta$ at the vertex $v \in\{x, y, z\}$ by $\alpha_{v}$.

First consider the case that $\Delta \notin F_{\rho}$ is no boundary triangle. We want to show that

$$
\begin{aligned}
E_{T_{\Delta}}(u)= & \frac{1}{2} \cot \alpha_{x}(u(y)-u(z))^{2}+\frac{1}{2} \cot \alpha_{z}(u(x)-u(y))^{2} \\
& +\frac{1}{2} \cot \alpha_{y}(u(z)-u(x))^{2} \\
\geqslant & \text { Const }_{\delta} \cdot\left|\frac{1}{2} \cot \alpha_{y}\right|(u(z)-u(x))^{2} .
\end{aligned}
$$

holds for some constant Const $\delta>0$. Thus we only need to consider the case $\alpha_{y}>$ $\pi / 2$. Take Const $\delta=1 /\left(\cot ^{2} \delta-1\right)$. As $\alpha_{x}, \alpha_{z}>\delta$ and $\alpha_{x}+\alpha_{y}+\alpha_{z}=\pi$, elementary calculations imply that

$0 \leqslant 1+$ Const $_{\delta} \cdot\left(1-\cot \alpha_{x} \cdot \cot \alpha_{z}\right)=\cot \alpha_{x} \cdot \cot \alpha_{z}+\cot \alpha_{y}\left(1+\right.$ Const $\left._{\delta}\right)\left(\cot \alpha_{x}+\cot \alpha_{z}\right)$.

This implies (10).

If $\Delta \in F_{\rho}$, we know that

$C_{[x, z]}=\cot \alpha_{y}^{E}+h \cdot r_{y}, \quad C_{[z, y]}=\cot \alpha_{x}^{E}+h \cdot r_{x}, \quad C_{[y, x]}=\cot \alpha_{z}^{E}+h \cdot r_{y}$,

where $\alpha_{v}^{E}$ denotes the angle at the vertex $v$ in the Euclidean triangle with vertices $x, y, z$ and $\left|r_{v}\right| \leqslant$ Const $_{\delta, \rho}$, see Appendix A.1. Therefore, there are constants const $_{\delta, \rho}, \widehat{\text { Const }}_{\delta, \rho}>0$ such that for all $0<h<$ const $_{\delta, \rho}$ we have $E_{T_{\Delta}}(u) \geqslant$ $\widetilde{\text { Const }}_{\delta, \rho}\left|C_{[x, z]}\right|(u(z)-u(x))^{2}$.

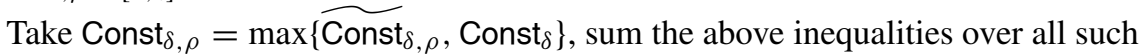
faces and deduce $E_{T^{\prime}}^{\prime}(u)-E_{T}(u) \leqslant$ Const $_{\delta, \rho} \cdot E_{T}(u)$. Now the claim follows.

\subsection{Convergence of Multi-valued Discrete Harmonic Functions and Discrete Holomorphic Integrals}

As a first step, we can deduce that the uniform limit of a sequence of discrete harmonic functions is harmonic. To this end, we say that a sequence of triangulated polygons $\left\{T_{n}\right\}$ approximates a domain $\Omega \subset \mathbb{C}$, if for $n \rightarrow \infty$ the following three quantities tend to zero: the maximal distance from a point of the boundary $\partial T_{n}$ to the 
set $\partial \Omega$, the maximal distance from a point of $\partial \Omega$ to the set $\partial T_{n}$, and the maximal edge length of the triangulation $T_{n}$.

Lemma 17 [7, Lemma 5.2] Let $\left\{T_{n}\right\}$ be a non-degenerate uniform sequence of Delaunay triangulations of polygons with boundary approximating a domain $\Omega$, such that no branch point is on $\partial \Omega$. Let $u_{n}: V_{n} \rightarrow \mathbb{R}$ be a sequence of discrete harmonic functions uniformly converging to a continuous function $u: \Omega \rightarrow \mathbb{R}$. Then the function $u: \Omega \rightarrow \mathbb{R}$ is harmonic.

Theorem 6 (Convergence of multi-valued discrete harmonic functions) Let $\left\{T_{n}\right\}$ be a non-degenerate uniform sequence of adapted Delaunay triangulations of $\mathscr{R}$ with maximal edge length $h_{n}$ tending to zero as $n \rightarrow \infty$. Let $z_{n} \in \widetilde{V}_{n}$ be a sequence of vertices converging to a point $z_{0} \in \widetilde{\mathscr{R}}$. Let $P_{n} \in \mathbb{R}^{2 g}$ be a sequence of vectors converging to a vector $P \in \mathbb{R}^{2 g}$. Then the functions $u_{T_{n}, P_{n}}: \widetilde{V}_{n} \rightarrow \mathbb{R}$ satisfying $u_{T_{n}, P_{n}}\left(z_{n}\right)=0$ converge to $u_{\mathscr{R}, P}: \widetilde{\mathscr{R}} \rightarrow \mathbb{R}$ with $u_{\mathscr{R}, P}\left(z_{0}\right)=0$ uniformly on every compact subset.

Proof We will start with some estimates on compact subsets of $\widetilde{\mathscr{R}}$ of a special form. Let $\pi=\operatorname{Pr}_{\mathscr{R}} \circ p: \widetilde{\mathscr{R}} \rightarrow \mathbb{C}$ be the local projection map $\operatorname{Pr}_{\mathscr{R}}$ composed with the universal covering $p$. For $v \in \widetilde{\mathscr{R}}$ denote by $\widetilde{B}_{r}(v) \subset \widetilde{\mathscr{R}}$ the subset which projects for $\pi(v) \in \mathbb{C}$ to an open intrinsic disc $B_{r}(\pi(v))=\pi\left(\widetilde{B}_{r}(v)\right)$ with radius $r$ about $\pi(v)$. If $\pi(v)=\infty$, we assume that $\pi\left(\widetilde{B}_{r}(v)\right)=\mathbb{C} \backslash B_{1 / r}(0)$. We restrict ourselves to the following cases:

$-\pi(v)=O$ is a branch point and $r=r_{O}(v)>0$ its associated radius defined in Section 2.3,

$-\pi(v) \in B_{\rho} \backslash \bigcup_{O \text { branch point }} B_{r_{O}}(O)$ and $r_{O}^{\min } / 8<r \leqslant r_{O}^{\min } / 2$, where $r_{O}^{\min } / 2:=$ $\min _{O \text { branch point }} r_{O} / 2$,

$-\pi(v)=\infty$ and $r=1 / \rho$.

Note that the union of these sets $\widetilde{B}_{r}(v)$ covers $\widetilde{R}$ and every compact set $K \subset \widetilde{\mathscr{R}}$ is contained in the union of finitely many of these sets.

Let $\widetilde{B}_{r}(v)$ be one of these sets. Consider those triangles of the given triangulation $\widetilde{T}_{n}$ which are completely contained in $\widetilde{B}_{r}(v)$ and denote by $\widetilde{T}_{n}(v, r)$ the connected component of these triangles which contains $v$. Choose $n_{1}$ such that for all $n>n_{1}$ the maximal edge length is sufficiently small, for example say $h_{n}<r_{O}^{\min } / 200$. Consider $u_{\widetilde{V}_{n}(v, r)}:=\left.u_{T_{n}, P_{n}}\right|_{\widetilde{V}_{n}(v, r)}$. By Lemma 16 and Corollary 1 the sequence of energies $E_{\widetilde{T}_{n}(v, r)}^{\prime}\left(u_{\widetilde{V}_{n}(v, r)}\right)$ is bounded. Thus the Equicontinuity Lemma 15 implies that the function $u_{\widetilde{V}_{n}(v, r)} \mid \widetilde{V}_{n} \cap \widetilde{B}_{\frac{3}{4} r}(v)$ has uniformly bounded differences. That is, there exists a constant Const $\mathscr{R}, P, \delta$ such that for all $n>n_{1}$ and $z, w \in \widetilde{V}_{n} \cap \widetilde{B}_{\frac{3}{4} r}(v)$ we have

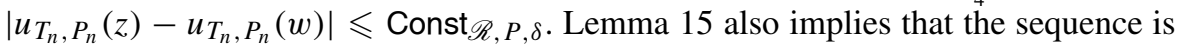
equicontinuous, that is, there exists a function $\delta(\varepsilon)$ for $\varepsilon>0$ such that for each $n>$ $n_{1}$ and $z, w \in \widetilde{V}_{n} \cap \widetilde{B}_{\frac{3}{4} r}(v)$ with $|z-w|<\delta(\varepsilon)$ we have $\left|u_{T_{n}, P_{n}}(z)-u_{T_{n}, P_{n}}(w)\right| \leqslant \varepsilon$. 
Now take a sequence of compact sets $K_{1} \subset K_{2} \subset \cdots \subset \widetilde{\mathscr{R}}$ such that $\widetilde{\mathscr{R}}=$ $\bigcup_{j=1}^{\infty} K_{j}$. Assume that $K_{1}$ contains all point of the convergent sequence $\left\{z_{n}\right\}$. Since $K_{1}$ is compact, it is contained in the union of finitely many of the sets considered above. Therefore, the sequence $\left.u_{T_{n}, P_{n}}\right|_{\widetilde{V}_{n} \cap K_{1}}$ is equicontinuous and has uniformly bounded differences (this bound also depends on $K_{1}$ ). Furthermore, as all $z_{n} \in K_{1}$ and $u_{T_{n}, P_{n}}\left(z_{n}\right)=0$, the sequence $\left.u_{T_{n}, P_{n}}\right|_{\widetilde{V}_{n} \cap K_{1}}$ is uniformly bounded. We deduce from the Arzelà-Ascoli theorem that there is a continuous function $u_{1}: K_{1} \rightarrow \mathbb{R}$ and a subsequence $\left\{l_{k}\right\}$ with $l_{1}=n_{1}$ such that $u_{T_{l_{k}}, P_{l_{k}}}$ converges to $u_{1}$ uniformly on $K_{1}$.

Analogously, we see that there is a continuous function $u_{1}: K_{1} \rightarrow \mathbb{R}$ and a subsequence $\left\{m_{k}\right\}$ of $\left\{l_{k}\right\}$ with $m_{1}=n_{1}, m_{2}=l_{2}$ such that $u_{T_{m_{k}}, P_{m_{k}}}$ converges to $u_{2}$ uniformly on $K_{2}$. Clearly, we have $u_{1}=u_{2}$ on $K_{1}$. This procedure can be continued and eventually we obtain a continuous function $u: \widetilde{R} \rightarrow \mathbb{R}$ and a subsequence $\left\{n_{k}\right\}$ of $\{1,2,3, \ldots\}$ such that $u_{T_{n_{k}}, P_{n_{k}}}$ converges uniformly to $u$ on each compact subset of $\widetilde{\mathscr{R}}$. Also, $u$ has the same periods $P$ as $u_{\mathscr{R}, P}$ and $u\left(z_{0}\right)=0$. Applying Lemma 17 to bounded domains not containing any branch point, we see that the limit function $u: \widetilde{R} \rightarrow \mathbb{R}$ is harmonic in $\widetilde{\mathscr{R}}$ except possibly at the branch points. But as $u$ is locally bounded, these singularities can be removed and therefore the continuous function $u$ is in fact harmonic on the whole surface $\widetilde{\mathscr{R}}$. Thus $u=u_{\mathscr{R}, P}$ by our normalization $u\left(z_{0}\right)=0=u_{\tilde{R}, P}\left(z_{0}\right)$.

Since the limit function $u=u_{\tilde{R}, P}$ is unique, it follows that the whole sequence $u_{T_{n}, P_{n}}$, not just the subsequence $u_{T_{n_{k}}, P_{n_{k}}}$, converges to $u_{\widetilde{R}, P}$ uniformly on every compact subset.

Proof of Theorem 4 Let $P_{n}, P \in \mathbb{R}^{2 g}$ be the periods of the real parts $\operatorname{Re} \phi_{T_{n}}^{l}: \widetilde{V} \rightarrow \mathbb{R}$ and $\operatorname{Re} \phi_{\mathscr{R}}^{l}: \widetilde{\mathscr{R}} \rightarrow \mathbb{R}$ of the discrete and smooth holomorphic integrals, respectively. Then by Theorem $5 \operatorname{Re} \phi_{T_{n}}^{l}=u_{T_{n}, P_{n}}$ and $\operatorname{Re} \phi_{\mathscr{R}}^{l}=u_{\widetilde{R}, P}$. Theorem 3, implies that $P_{n} \rightarrow P$ as $n \rightarrow \infty$. Thus we deduce from Theorem 6 that the real parts $\operatorname{Re} \phi_{T_{n}}^{l}$ converge to $\operatorname{Re} \phi_{\mathscr{R}}^{l}$ uniformly on every compact subset. Convergence of the imaginary parts is proven analogously due to the following Lemma 18.

Lemma 18 (Conjugate Functions Principle) Let $f=(\operatorname{Re} f: \widetilde{V} \rightarrow \mathbb{R}, \operatorname{Im} f: \widetilde{F} \rightarrow$ $\mathbb{R})$ be a discrete holomorphic integral. Then $E_{T}(\operatorname{Re} f)=E_{T}(\operatorname{Imf})$.

Proof This follows immediately from (5) together with the definitions of the discrete energies in (3) and (6).

\section{Improved Convergences of Period Matrices and Holomorphic Integrals for Polyhedral Surfaces}

The techniques applied for adapted triangulations near branch points may also be used to improve the order of convergence of period matrices and holomorphic integrals for polyhedral surfaces compared to the results obtained in [7]. A polyhedral surface $\mathscr{S}$ is an oriented two-dimensional manifold without boundary which has a piecewise flat metric with isolated conical singularities. An example is the surface of 
a polyhedron in three-dimensional space. Let $T_{\mathscr{S}}$ be a geodesic triangulation of the polyhedral surface $\mathscr{S}$ such that all faces are flat triangles. Note in particular, that all singular points of the metric are vertices of $T_{\mathscr{S}}$. On all edges we use cotan weights given by (1).

If $\gamma_{O}>1 / 2$, we do not adapt the triangulation further. But for singularities $O$ with $\gamma_{O} \leqslant 1 / 2$ we consider a chart $g_{O}$, which maps a neighborhood $\mathscr{C}_{O}$ of $O$ to a neighborhood of the origin in $\mathbb{C}$. Furthermore, we can introduce as above "polar coordinates" $(r, \phi)$ on $\mathscr{C}_{O}$ with the origin at the vertex $O$. We map all vertices in $\mathscr{C}_{O}$ to a neighborhood of the origin in $\mathbb{C}$ by the chart $g_{O}: \mathscr{C}_{O} \rightarrow \mathbb{C}, g_{O}(r, \phi):=$ $r^{\gamma O} \mathrm{e}^{i \gamma_{O} \phi}$. If $\gamma_{O} \leqslant 1 / 2$ we demand that the images of any two incident vertices in $\mathscr{C}_{O}$ have maximum distance $h$. Consider any triangle $\Delta$ in $\mathscr{C}_{O}$ whose vertex $z$ nearest to $O$ satisfies $|O z| \geqslant h_{O}=h^{1 /\left(2 \gamma_{O}\right)}$, where $|O z|$ denotes the distance of $z$ to $O$ in $\mathscr{S}$. As in Section 2.3 we deduce from our assumption that the maximal edge length in $\Delta$ is smaller than $h \cdot|O z|^{1-\gamma_{O}}$.

Applying the estimates of Sections 3.1 and 3.2, we obtain the following improved versions of Theorems 2.5 and 2.7 of [7].

Theorem 7 (Energy convergence) For each $\delta>0$ and each smooth multi-valued harmonic function $u: \widetilde{\mathscr{S}} \rightarrow \mathbb{R}$ there are two constants Const $_{u, \delta, \mathscr{S}}$, const $_{u, \delta, \mathscr{S}}>$ 0 such that for any adapted triangulation $T$ of $\mathscr{S}$ with maximal edge length $h<$ const $_{u, \delta, \mathscr{S}}$ and minimal face angle $>\delta$ we have

$$
\left|E_{T}\left(\left.u\right|_{\tilde{V}}\right)-E(u)\right| \leqslant \text { Const }_{u, \delta, \mathscr{S}} \cdot h .
$$

Theorem 8 (Convergence of discrete period matrices) For each $\delta>0$ there exist constants Const $_{\delta, \mathscr{S}}$, const $_{\delta, \mathscr{S}}>0$ such that for any adapted triangulation $T$ of $\mathscr{S}$ with maximal edge length $h<$ const $_{u, \delta, \mathscr{S}}$ and minimal face angle $>\delta$ we have

$$
\left\|\Pi_{T}-\Pi_{\mathscr{S}}\right\| \leqslant \text { Const }_{\delta, \mathscr{S}} \cdot h .
$$

Theorem 9 (Convergence of discrete holomorphic integrals) Let $\left\{T_{n}\right\}$ be a sequence of non-degenerate uniform adapted triangulations of $\mathscr{S}$ with maximal edge length $\underline{h}_{n} \rightarrow 0$ as $n \rightarrow \infty$. Let $\pi: \widetilde{\mathscr{S}} \rightarrow S$ be the universal covering of $\mathscr{S}$. Denote by $\widetilde{T}_{n}$ the corresponding triangulation of $\widetilde{\mathscr{S}}$ such that $\pi\left(\widetilde{T}_{n}\right)=T_{n}$. Let $z_{n} \in \widetilde{V}_{n}$ be a sequence of vertices converging to a point $z_{0} \in \tilde{\mathscr{S}}$. Let $\Delta_{n} \in \widetilde{F}_{n}$ be a sequence of faces with its vertices converging to $z_{0}$. Then for each $1 \leqq l \leqslant g$ the discrete holomorphic integrals $\phi_{T}^{l}=\left(\operatorname{Re} \phi_{T}^{l}: \widetilde{V} \rightarrow \mathbb{R}, \operatorname{Im} \phi_{T}^{l}: \widetilde{F} \rightarrow \mathbb{R}\right)$ normalized at $z_{n}$ and $w_{n}$ converge uniformly on each compact set to the holomorphic integral $\phi_{\mathscr{S}}^{l}: \widetilde{\mathscr{S}} \rightarrow \mathbb{C}$ normalized at $z_{0}$

\section{Numerical Experiments}

In the following, we present some numerical analysis for our convergence results detailed above. We are very grateful to Stefan Sechelmann for writing software and performing numerical experiments. 
Mainly, we apply the scheme described in Section 2, but with some small changes. In particular, we could use stereographic projection from the sphere $\mathbb{S}^{2} \cong \hat{\mathbb{C}}$ to $\mathbb{C}$ from the north pole $(\infty)$ and the south pole (0) resp. as our charts $\operatorname{Pr}_{\mathscr{R}}$ and $\operatorname{Pr}_{\mathscr{R}}^{\infty}$. We could then work with Euclidean triangles in the plane $\mathbb{C}$ as in the previous sections or with their preimages on $\mathbb{S}^{2} \cong \hat{\mathbb{C}}$. For practical reasons, we do not work with these triangles in $\mathbb{S}^{2} \subset \mathbb{R}^{3}$. Instead, we take the vertices and add straight line segments in $\mathbb{R}^{3}$ between incident vertices. For every original triangle $\Delta$ on $\mathbb{S}^{2}$ we obtain a corresponding triangle $\Delta^{S}$ in $\mathbb{R}^{3}$, see Fig. 2 for some examples of triangulations.

Furthermore, we use an approximation of the discrete energy $E_{T}$ and of the discrete multi-valued harmonic functions $u_{T, P}$ because we use slightly different weights instead of those given in Section 2.1. As $\rho$ is fixed and the maximum edge length $h$ tends to zero, any angle $\alpha$ in the triangle $\Delta$ and the corresponding angle $\alpha^{S}$ in the triangle $\Delta^{S}$ only differ by an error of order $h$, in particular $\left|\alpha-\alpha^{S}\right| \leqslant \operatorname{Const}_{\delta, \rho} \cdot l_{\max }(\Delta)$. Thus, for uniform Delaunay triangulations which we consider, the weights $c^{S}(e)=$ $\frac{1}{2}\left(\cot \alpha_{e}^{S}+\cot \beta_{e}^{S}\right)$, using the cotan-formula for the angles of the triangles $\Delta^{S}$, can be estimated using the original weights $c(e)$ for $T$. More precisely, there is a constant Const $_{\delta, \rho}$ such that $c(e) \cdot\left(1-\right.$ Const $\left._{\delta, \rho} \cdot h\right) \leqslant c^{S}(e) \leqslant c(e) \cdot\left(1+\right.$ Const $\left._{\delta, \rho} \cdot h\right)$. This implies $E_{T}(u) \cdot\left(1-\right.$ Const $\left._{\delta, \rho} \cdot h\right) \leqslant E_{T}^{S}(u) \leqslant E_{T}(u) \cdot\left(1+\right.$ Const $\left._{\delta, \rho} \cdot h\right)$. Thus for $0<h<1$ Const $_{\delta, \rho}$ and some constant Const $_{P, \delta, \mathscr{R}, \rho}>0$ we obtain similarly as in the proof of Lemma 13 that

$$
\left|E_{T}^{S}\left(u_{T, P}^{S}\right)-E\left(u_{\mathscr{R}, P}\right)\right| \leqslant \operatorname{Const}_{P, \delta, \mathscr{R}, \rho} \cdot h .
$$

As concrete examples we consider two surfaces with known period matrices, namely the torus $\mathscr{T}$ of genus 1 with branch points $0.5+0.4 i,-0.3+0.2 i,-0.1$, $0.1-0.2 i$ and Lawson's minimal surface $\mathscr{L}$ of genus 2 which corresponds to the hyperelliptic curve $\mu^{2}=\lambda^{6}-1$ with branch points $\mathrm{e}^{i k \pi / 3}, k=0,1, \ldots, 5$. The smooth period matrices are $\Pi_{\mathscr{T}} \approx 0.175+1.017 i$ and $\Pi_{\mathscr{L}}=\frac{i}{\sqrt{3}}\left(\begin{array}{cc}2 & -1 \\ -1 & 2\end{array}\right)$.

We compare four different types of triangulations of $\mathbb{S}^{2}$ which are used as basis for the computations of the discrete period matrices. Figure 2 shows an example for each of these four types. In order to simplify calculations and the construction of cycles, we always use the same triangulation on every sheet of the covering.

Random: We sample points at random on the sphere and then build the corresponding Delaunay triangulation.
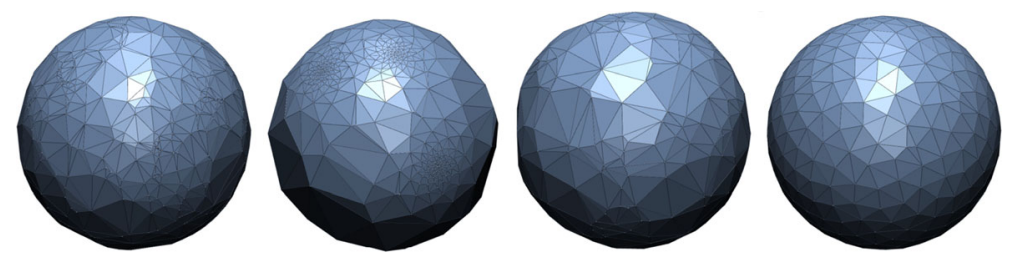

Fig. 2 Examples of our four types of triangulations used for the torus $\mathscr{T}$ : Random with adapted triangles (Adapted Random o), Homogeneous with adapted triangles (Adapted Homogeneous $\square$ ), Random without adapted triangles (Random $\diamond$ ), Homogeneous without adapted triangles (Homogeneous $\triangle$ 
Homogeneous: The points on a sphere are evenly distributed by means of a Fibonacci spiral. This leads to very 'regular' or 'homogeneous' triangulations with triangles which are almost equilateral, except near branch points, which are in general additional vertices.

These two types of triangulations are directly used for further computations (called Random $\diamond$ and Homogeneous $\triangle$ ). In these two cases the estimates of [7] apply. Our numerical results are plotted in the lower rows of Figs. 3 and 4. The log-log plots show that the error behaves indeed like $\sqrt{h}$ for 'Random', where $h$ is the maximal edge length. This was also observed in the example studied in Section 7.3 of [7]. Nevertheless, for the more regular triangulations using Fibonacci spirals (Homogeneous), our numerical evidence indicates a higher order of the error bound, possibly a linear dependence on $h$.

According to our new idea of adapted triangulations explained in Section 2.3, we refine the examples of the two types of triangulations above in a neighborhood of the branch points by suitably adding vertices (called Adapted Random o and Adapted Homogeneous $\square$ ). Our results in Figs. 3 and 4 confirm that the error between smooth and discrete period matrices decreases indeed faster in the adapted case. In particular, the log-log plots in the upper row of Figs. 3 and 4, respectively, show that for our adapted method the error depends at least linearly on the maximal edge length $h$ (as
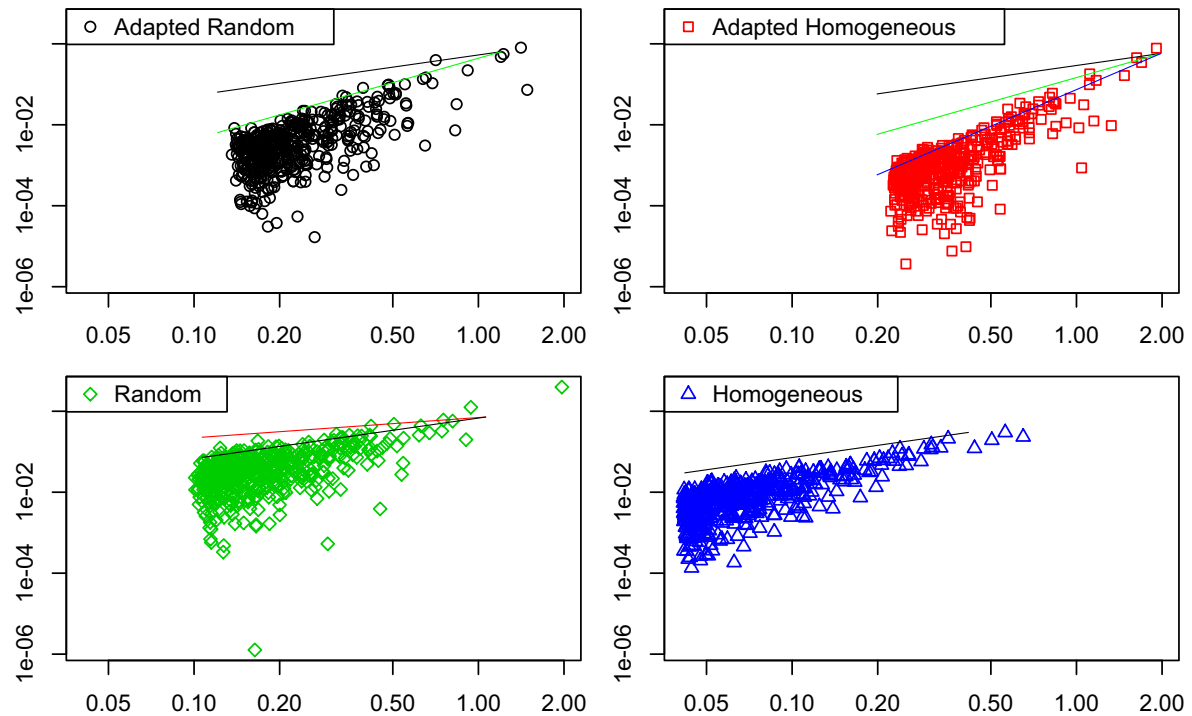

Fig. 3 For a variety of examples - for each of the four different types of triangulations respectively - the plots show the approximation error for the period matrices of the torus $\mathscr{T}$ plotted over the maximal edge length of the triangulation. We have added some lines to the log-log-plots. The black line (in all plots) has slope 1 and corresponds to an error estimate of order $h$. This is our estimate for the adapted case as shown in Theorem 3. The green line has slope 2 (order $h^{2}$ ), the blue line has slope 3 (order $h^{3}$ ), and the red line has slope $1 / 2$ (order $\sqrt{h}$, proven in [7]). Our results are scattered, as we only control the maximal edge length, but no other parameter like for example the minimal angle in the triangles. The plots for the Homogeneous case (adapted and non-adapted) suggest that the order of the error in terms of the maximal edge length increases for these more regular triangulations 

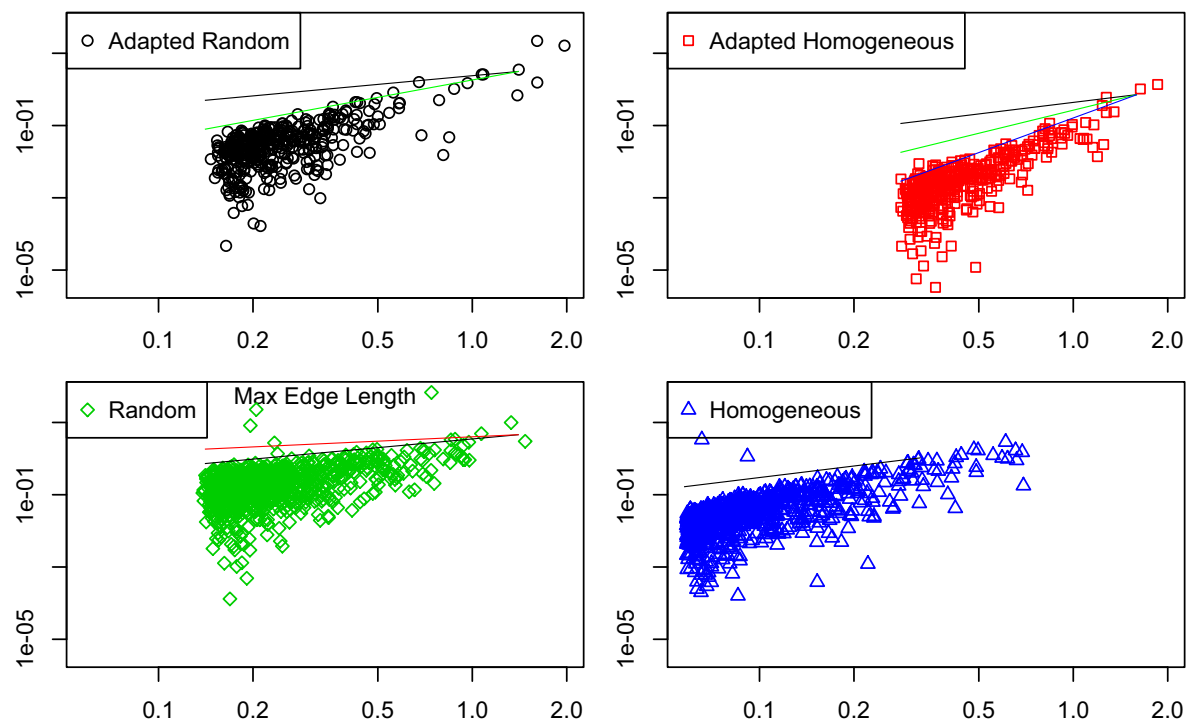

Fig. 4 For a variety of examples - for each of the four different types of triangulations respectively - the plots show the approximation error for the period matrices of Lawson's minimal surface $\mathscr{L}$ of genus 2 plotted over the maximal edge length of the triangulation. We have added some lines to the log-log-plots. The black line (in all plots) has slope 1 and corresponds an estimate of order $h$. This is our estimate in the adapted case as shown in Theorem 3 . The green line has slope 2 (order $h^{2}$ ), the blue line has slope 3 , and the red line has slope $1 / 2$ (order $\sqrt{h}$, proven in [7]). Our results are scattered, as we do only control the maximal edge length and no other parameter like for example the minimal angle in the triangles. This may also explain the upward outliers in the plots in the non-adapted cases (Random and Homogeneous). The plots for the Homogeneous case (adapted and non-adapted) suggest that the order of the error is terms of the maximal edge length increases for these more regular triangulations

proven in Theorem 3) and is again probably even of higher order for more regular triangulations using Fibonacci spirals.

Recall that the actual bounds on the approximation error also depend on the angles in the triangles which differ for all examples we computed. This is one reason why our numerical results scatter within some domain and even include some outliers like in the Random and Homogeneous case for Lawson's minimal surface $\mathscr{L}$ in Fig. 4. In our proof we only use some (rough) estimate of the angles such that our constants in Theorems 3 and 13 depend only on the minimal angle of the adapted triangulation. We did not study the dependence of the angles in detail, but our numerical results suggest that the order of convergence also depends significantly on further parameters of the triangulations, as regularity and the distribution of angles in the triangles.

\section{Appendix A.1: Interpolation Function on Boundary Triangles and Estimates on Corresponding Edge Weights}

In the following, we expose the calculations for the energy of the interpolation function and the corresponding edge weights. 
Let $\Delta[x, y, z] \in F_{\rho}$ be a boundary triangle. Without loss of generality, we assume that the vertices are labelled such that $x \in B_{\rho}(0)$ and $y, z \in \mathbb{C} \backslash B_{\rho}(0)$. Therefore, the triangle $\Delta[x, y, z]$ is bounded by two straight edges $[x, y]$ and $[x, z]$ and by the trace of the curve $s:[0,1] \rightarrow \mathbb{C}, s(t)=\frac{y z}{z+t(y-z)}$, connecting $y$ and $z$ which is in general a circular arc. We parametrize this triangle by

$$
p:[0,1] \times[0,1] \rightarrow \Delta[x, y, z], \quad p(\tau, \sigma)=x+\sigma(s(\tau)-x) .
$$

Note that $p$ is bijective for $\sigma \neq 0$. In this parametrization, the interpolation function is $u_{\Delta}(\tau, \sigma):=I_{T} u(p(\tau, \sigma))=u_{x}+\sigma\left(u_{y}-u_{x}+\tau\left(u_{z}-u_{y}\right)\right)$ as explained in Section 2.1. Here we use the notation $u_{v}=u(v)$ for the values of the given smooth function $u$ at the vertices $x, y, z$. Therefore, we obtain

$$
\begin{aligned}
& \int_{\Delta[x, y, z]}\left|\nabla I_{T} u\right|^{2}=\int_{0}^{1} \int_{0}^{1} D u_{\Delta}(\tau, \sigma) D p^{-1}(p(\tau, \sigma))\left(D p^{-1}(p(\tau, \sigma))\right)^{T}\left(D u_{\Delta}(\tau, \sigma)\right)^{T}|\operatorname{det} D p(\tau, \sigma)| d \tau d \sigma \\
& =\int_{0}^{1} \int_{0}^{1}\left(\left(u_{z}-u_{y}\right)^{2} \frac{|s(\tau)-x|^{2}-2 \tau \operatorname{Re}\left((s(\tau)-x) \overline{s^{\prime}(\tau)}\right)+\tau^{2}\left|s^{\prime}(\tau)\right|^{2}}{\left|\operatorname{Im}\left((s(\tau)-x) \overline{s^{\prime}(\tau)}\right)\right|^{2}}\right. \\
& +\left(u_{y}-u_{x}\right)^{2} \frac{\left|s^{\prime}(\tau)\right|^{2}}{\left|\operatorname{Im}\left((s(\tau)-x) \overline{s^{\prime}(\tau)}\right)\right|^{2}} \\
& \left.+\left(u_{y} v-u_{x}\right)\left(u_{z}-u_{y}\right) \frac{2 \tau\left|s^{\prime}(\tau)\right|^{2}-2 \operatorname{Re}\left((s(\tau)-x) \overline{\left.s^{\prime}(\tau)\right)}\right.}{\left|\operatorname{Im}\left((s(\tau)-x) \overline{s^{\prime}(\tau)}\right)\right|^{2}}\right)|\operatorname{det} D p(\tau, \sigma)| d \tau d \sigma \\
& =\frac{1}{2} \int_{0}^{1}\left(\left(u_{z}-u_{y}\right)^{2} \frac{|s(\tau)-x|^{2}-2 \tau \operatorname{Re}\left((s(\tau)-x) \overline{s^{\prime}(\tau)}\right)+\tau^{2}\left|s^{\prime}(\tau)\right|^{2}}{\left|\operatorname{Im}\left((s(\tau)-x) \overline{s^{\prime}(\tau)}\right)\right|}\right. \\
& +\left(u_{y}-u_{x}\right)^{2} \frac{\left|s^{\prime}(\tau)\right|^{2}}{\left|\operatorname{Im}\left((s(\tau)-x) \overline{s^{\prime}(\tau)}\right)\right|} \\
& \left.+\left(u_{y}-u_{x}\right)\left(u_{z}-u_{y}\right) \frac{2 \tau\left|s^{\prime}(\tau)\right|^{2}-2 \operatorname{Re}\left((s(\tau)-x) \overline{s^{\prime}(\tau)}\right)}{\left|\operatorname{Im}\left((s(\tau)-x) \overline{s^{\prime}(\tau)}\right)\right|}\right) d \tau,
\end{aligned}
$$

as $D u_{\Delta}(\tau, \sigma)=\left(\sigma\left(u_{z}-u_{y}\right), u_{y}-u_{x}+\tau\left(u_{z}-u_{y}\right)\right), D p(\tau, \sigma)=$ $\left(\begin{array}{l}\sigma \operatorname{Re}\left(s^{\prime}(\tau)\right) \operatorname{Re}(s(\tau)-x) \\ \sigma \operatorname{Im}\left(s^{\prime}(\tau)\right) \operatorname{Im}(s(\tau)-x)\end{array}\right)$, and $\operatorname{det}(D p(\tau, \sigma))=\sigma \operatorname{Im}\left((s(\tau)-x) \overline{s^{\prime}(\tau)}\right)$. Thus we deduce that

$$
\int_{\Delta[x, y, z]}\left|\nabla I_{T} u\right|^{2}=C_{[x, y]}(u(x)-u(y))^{2}+C_{[y, z]}(u(y)-u(z))^{2}+C_{[z, x]}(u(z)-u(x))^{2},
$$

$$
\text { where } \quad \begin{aligned}
C_{[x, y]} & =\frac{1}{2} \int_{0}^{1} \frac{(1-\tau)\left|s^{\prime}(\tau)\right|^{2}+\operatorname{Re}\left((s(\tau)-x) \overline{s^{\prime}(\tau)}\right)}{\left|\operatorname{Im}\left((s(\tau)-x) \overline{s^{\prime}(\tau)}\right)\right|} d \tau, \\
C_{[y, z]} & \left.=\frac{1}{2} \int_{0}^{1} \frac{|s(\tau)-x|^{2}+\tau(\tau-1)\left|s^{\prime}(\tau)\right|^{2}+\operatorname{Re}\left((s(\tau)-x) \overline{s^{\prime}(\tau)}\right)}{\left|\operatorname{Im}\left((s(\tau)-x) \overline{s^{\prime}(\tau)}\right)\right|} d \tau, \overline{s^{\prime}(\tau)}\right) \\
C_{[z, x]} & =\frac{1}{2} \int_{0}^{1} \frac{\tau\left|s^{\prime}(\tau)\right|^{2}-\operatorname{Re}((s(\tau)-x .}{\left|\operatorname{Im}\left((s(\tau)-x) \overline{s^{\prime}(\tau)}\right)\right|} d \tau
\end{aligned}
$$

This gives an explicit way to calculate the edge weights. Note that by the same method we can obtain the usual cotan-weights on the Euclidean triangle with vertices 
$x, y, z$, if we use $s_{E}(t)=y+t(z-y)$ instead of $s(t)$ for $t \in[0,1]$. The function $s_{E}$ is the usual linear parametrization of the straight edge from $y$ to $z$.

An important observation is that these seemingly 'complicated' weights are in fact only small perturbations of the usual cotan-weights if the edge length is small enough. To see this, we will estimate the quantities in the above integrals compared to the corresponding quantities for $s_{E}$.

Proof of the estimate in Remark 1 We assume that there is some $\delta>0$ such that all angles in the triangle $\Delta[x, y, z]$ are in $[\delta, \pi-\delta]$ and also all angles of the Euclidean triangle with vertices $x, y, z$ are in $[\delta, \pi-\delta]$. In the following, we will always assume that $\tau \in[0,1]$ as in the integral terms above. Also, we are not interested in the best possible estimates, any constant, depending only on the indicated parameters, will suffice.

First note that $\left|\operatorname{Im}\left((s(\tau)-x) \overline{s^{\prime}(\tau)}\right)\right| \geqslant|s(\tau)-x|\left|s^{\prime}(\tau)\right| \sin \delta$ and $\mid \operatorname{Im}\left(\left(s_{E}(\tau)-\right.\right.$ $\left.x) \overline{s_{E}^{\prime}(\tau)}\right)|\geqslant| s_{E}(\tau)-x|| z-y \mid \sin \delta$. Denote by $h$ the maximal edge length of $\Delta[x, y, z]$, so $h \geqslant \max \{|y-x|,|z-x|$, length $(s)\}$. As $\Delta[x, y, z]$ is a boundary triangle, we have

$$
\frac{\rho^{2}}{(\rho+h)^{2}} \leqslant\left|\frac{s^{\prime}(\tau)}{s_{E}(\tau)}\right|=\frac{\left|s^{\prime}(\tau)\right|}{|y-z|} \leqslant \frac{(\rho+h)^{2}}{\rho^{2}} .
$$

Furthermore, we deduce that $1-$ Const $_{\delta, \rho} \cdot h \leqslant\left|\frac{s(\tau)-x}{s_{E}(\tau)-x}\right| \leqslant 1+$ Const $_{\delta, \rho} \cdot h$ as

$$
\left|\frac{s(\tau)-s_{E}(\tau)}{s_{E}(\tau)-x}\right|=\left|\frac{\tau(\tau-1)(y-z)^{2}}{z+\tau(y-z)} \cdot \frac{1}{(y-x)+\tau(z-y)}\right| \leqslant \frac{h}{\rho \sin ^{2} \delta} .
$$

Further, note that using the sine law

$$
\sin ^{2} \delta \leqslant \frac{\left|s_{E}(\tau)-x\right|}{\left|s_{E}^{\prime}(\tau)\right|}=\frac{|(y-x)+\tau(z-y)|}{|y-z|} \leqslant 1+\frac{1}{\sin \delta} .
$$

Combining these estimates, we have

$$
\begin{aligned}
& \frac{\left|s(\tau)-s_{E}(\tau)\right|\left|s^{\prime}(\tau)\right|}{\left|\operatorname{Im}\left(\left(s_{E}(\tau)-x\right) \overline{s_{E}^{\prime}(\tau)}\right)\right|} \leqslant \frac{\left|s(\tau)-s_{E}(\tau)\right|}{\left|s_{E}(\tau)-x\right|} \cdot\left|\frac{s^{\prime}(\tau)}{s_{E}(\tau)}\right| \cdot \frac{1}{\sin \delta} \leqslant \text { Const }_{\delta, \rho} \cdot h, \\
& \frac{\left|s^{\prime}(\tau)\right|^{2}}{\left|\operatorname{Im}\left(\left(s_{E}(\tau)-x\right) \overline{s_{E}^{\prime}(\tau)}\right)\right|} \leqslant\left|\frac{s^{\prime}(\tau)}{s_{E}(\tau)}\right| \cdot \frac{\left|s_{E}^{\prime}(\tau)\right|^{2}}{\left|s_{E}^{\prime}(\tau)\right|\left|s_{E}(\tau)-x\right| \sin \delta} \leqslant \text { Const }_{\delta, \rho} \cdot h, \\
& \frac{|s(\tau)-x|^{2}}{\left|\operatorname{Im}\left(\left(s_{E}(\tau)-x\right) \overline{s_{E}^{\prime}(\tau)}\right)\right|} \leqslant \frac{|s(\tau)-x|^{2}}{\left|s_{E}(\tau)-x\right|^{2}} \cdot \frac{\left|s_{E}(\tau)-x\right|^{2}}{\left|s_{E}^{\prime}(\tau)\right|\left|s_{E}(\tau)-x\right| \sin \delta} \leqslant \text { Const }_{\delta, \rho} \cdot h .
\end{aligned}
$$

Furthermore, as $h \leqslant \rho / 2$,

$$
\frac{\left|s_{E}(\tau)-x\right|\left|s^{\prime}(\tau)-s_{E}^{\prime}(\tau)\right|}{\left|\operatorname{Im}\left(\left(s_{E}(\tau)-x\right) \overline{s_{E}^{\prime}(\tau)}\right)\right|} \leqslant \frac{\left|s^{\prime}(\tau)-s_{E}^{\prime}(\tau)\right|}{|y-z| \sin \delta}=\frac{|y-z|\left|\tau^{2}(y-z)^{2}+2 \tau z-z\right|}{|z+\tau(y-z)|^{2} \sin \delta} \leqslant \frac{6}{\rho \sin \delta} \cdot h .
$$


Therefore, we obtain

$$
\begin{aligned}
& \left|\frac{\operatorname{Im}\left((s(\tau)-x) \overline{s^{\prime}(\tau)}\right)}{\operatorname{Im}\left(\left(s_{E}(\tau)-x\right) \overline{s_{E}^{\prime}(\tau)}\right)}-1\right|=\mid \frac{\operatorname{Im}\left(\left(s(\tau)-s_{E}(\tau)\right) \overline{s^{\prime}(\tau)}\right)}{\operatorname{Im}\left(\left(s_{E}(\tau)-x\right) \overline{s_{E}^{\prime}(\tau)}\right)} \\
& +\frac{\operatorname{Im}\left((s(\tau)-x) \overline{\left(s^{\prime}(\tau)-s_{E}^{\prime}(\tau)\right)}\right)}{\operatorname{Im}\left(\left(s_{E}(\tau)-x\right) \overline{s_{E}^{\prime}(\tau)}\right)} \mid \leqslant \text { Const }_{\rho, \delta} \cdot h \\
& \left|\frac{\operatorname{Re}\left((s(\tau)-x) \overline{s^{\prime}(\tau)}\right)-\operatorname{Re}\left(\left(s_{E}(\tau)-x\right) \overline{s_{E}^{\prime}(\tau)}\right)}{\operatorname{Im}\left(\left(s_{E}(\tau)-x\right) \overline{s_{E}^{\prime}(\tau)}\right)}\right|=\mid \frac{\operatorname{Re}\left(\left(s(\tau)-s_{E}(\tau)\right) \overline{s^{\prime}(\tau)}\right)}{\operatorname{Im}\left(\left(s_{E}(\tau)-x\right) \overline{s_{E}^{\prime}(\tau)}\right)} \\
& +\frac{\operatorname{Re}\left(\left(s_{E}(\tau)-x\right) \overline{\left(s^{\prime}(\tau)-s_{E}^{\prime}(\tau)\right)}\right)}{\operatorname{Im}\left(\left(s_{E}(\tau)-x\right) \overline{s_{E}^{\prime}(\tau)}\right)} \mid \leqslant \operatorname{Const}_{\rho, \delta}^{\prime} \cdot h
\end{aligned}
$$

This also implies $\left|\frac{\operatorname{Re}\left((s(\tau)-x) \overline{s^{\prime}(\tau)}\right)}{\operatorname{Im}\left(\left(s_{E}(\tau)-x\right) \overline{\left.s_{E}^{\prime}(\tau)\right)}\right.}\right| \leqslant\left|\frac{\operatorname{Re}\left(\left(s_{E}(\tau)-x\right) \overline{\left.s_{E}^{\prime}(\tau)\right)}\right.}{\operatorname{Im}\left(\left(s_{E}(\tau)-x\right) \overline{\left.s_{E}^{\prime}(\tau)\right)}\right.}\right|+$ Const $_{\rho, \delta}^{\prime} \cdot h \leqslant$ Const $_{\rho, \delta}^{\prime \prime}$ Denote by $\alpha_{z}^{E} \in[\delta, \pi-\delta]$ the angle in the Euclidean triangle with vertices $x, y, z$. Then the previous estimates imply

$$
\begin{aligned}
& \left|C_{[x, y]}-\frac{1}{2} \cot \alpha_{z}^{E}\right| \leqslant \frac{1}{2} \int_{0}^{1}\left(\frac{(1-\tau)\left(\left|s^{\prime}(\tau)\right|^{2}\left|\frac{\operatorname{Im}\left((s(\tau)-x) \overline{s^{\prime}(\tau)}\right)}{\operatorname{Im}\left(\left(s_{E}(\tau)-x\right) \overline{\left.s_{E}^{\prime}(\tau)\right)}\right.}-1\right|+\left|\frac{\left|s^{\prime}(\tau)\right|^{2}}{\left|s_{E}^{\prime}(\tau)\right|^{2}}-1\right|\left|s_{E}^{\prime}(\tau)\right|^{2}\right)}{\left|\operatorname{Im}\left(\left(s_{E}(\tau)-x\right) \overline{s_{E}^{\prime}(\tau)}\right)\right|}\right. \\
& +\frac{\left|\operatorname{Re}\left((s(\tau)-x) \overline{s^{\prime}(\tau)}\right)\right|\left|\frac{\operatorname{Im}\left((s(\tau)-x) \overline{s^{\prime}(\tau)}\right)}{\operatorname{Im}\left(\left(s_{E}(\tau)-x\right) \overline{\left.s_{E}^{\prime}(\tau)\right)}\right.}-1\right|}{\left|\operatorname{Im}\left(\left(s_{E}(\tau)-x\right) \overline{s_{E}^{\prime}(\tau)}\right)\right|} \\
& \left.+\frac{\left|\operatorname{Re}\left((s(\tau)-x) \overline{s^{\prime}(\tau)}\right)-\operatorname{Re}\left(\left(s_{E}(\tau)-x\right) \overline{s_{E}^{\prime}(\tau)}\right)\right|}{\left|\operatorname{Im}\left(\left(s_{E}(\tau)-x\right) \overline{s_{E}^{\prime}(\tau)}\right)\right|}\right) d \tau \\
& \leqslant \text { Const }_{\rho, \delta}^{\prime \prime \prime} \cdot h .
\end{aligned}
$$

Similarly, we can deduce that $\left|C_{[y, z]}-\frac{1}{2} \cot \alpha_{x}^{E}\right| \leqslant$ Const $_{\rho, \delta}^{\prime \prime \prime} \cdot h$ and $\mid C_{[z, x]}-$ $\frac{1}{2} \cot \alpha_{y}^{E} \mid \leqslant$ Const $_{\rho, \delta}^{\prime \prime \prime} \cdot h$.

\section{Appendix A.2: Proof of Lemma 11}

Note that as $u$ is smooth, there exist constants Const $_{u, \rho}$, const $_{u, \rho}>0$ such that for $0<h<$ const $_{u, \rho}$ we have $|u(x)-u(y)|^{2} /|x-y|^{2} \leqslant$ Const $_{\delta, \rho} \cdot \max _{w \in \Delta}\left\|D^{1} u(w)\right\|^{2} \leqslant$ Const $_{u, \rho}$ for all edges $e=[x, y]$ of boundary triangles in $F_{\rho}$ with edge lengths smaller than $h$.

Using our estimates of Appendix A.1 we deduce that there exists a constant Const $_{\delta, \rho}$ such that under our assumptions on angles and edge lengths we have the 
following estimates: For every boundary triangle $\Delta[x, y, z] \in F_{\rho}$ and with the notation of Appendix A.1

$$
\begin{aligned}
|z-y|^{2} \frac{|s(\tau)-x|^{2}}{\left|\operatorname{Im}\left((s(\tau)-x) \overline{s^{\prime}(\tau)}\right)\right|^{2}} & \leqslant \text { Const }_{\delta, \rho}, \quad|y-x|^{2} \frac{\left|s^{\prime}(\tau)\right|^{2}}{\left|\operatorname{Im}\left((s(\tau)-x) \overline{s^{\prime}(\tau)}\right)\right|^{2}} \\
& \leqslant \text { Const }_{\delta, \rho},|y-x||z-y| \frac{\left|\operatorname{Re}\left((s(\tau)-x) \overline{s^{\prime}(\tau)}\right)\right|}{\left|\operatorname{Im}\left((s(\tau)-x) \overline{s^{\prime}(\tau)}\right)\right|^{2}} \\
& \leqslant \text { Const }_{\delta, \rho} .
\end{aligned}
$$

Now formula (12) leads to the estimate

$$
\int_{\Delta[x, y, z]}\left|\nabla I_{T} u\right|^{2} \leqslant \operatorname{Const}_{\delta, \rho, u} \cdot \operatorname{Area}(\Delta[x, y, z]),
$$

where $\operatorname{Const}_{\delta, \rho, u} \leqslant$ Const $_{\delta, \rho} \cdot \max _{w \in \Delta}\left\|D^{1} u(w)\right\|^{2}$. Summing up these energies, we obtain

$$
\begin{aligned}
E_{F_{\rho}}(u) & =\sum_{\Delta \in F_{\rho}} E_{T_{\Delta}}(u)=\sum_{\Delta \in F_{\rho}} \int_{\Delta[x, y, z]}\left|\nabla I_{T} u\right|^{2} \leqslant \sum_{\Delta \in F_{\rho}} \operatorname{Const}_{\delta, \rho, u} \cdot \operatorname{Area}(\Delta[x, y, z]) \\
& \leqslant \text { Const }_{\delta, \rho, u} \cdot \operatorname{Area}\left(B_{\rho+h}(0) \backslash B_{\rho-h}(0)\right) \leqslant \operatorname{Const}_{\delta, \rho, u} \cdot 4 h \rho \cdot d \leqslant \text { Const }_{u, \delta, \rho, \mathscr{R}} \cdot h,
\end{aligned}
$$

where $d$ denotes the degree of the covering map for $\mathscr{R}$.

\section{Appendix A.3: Proof of Equicontinuity Lemma 15}

First note that condition (D) from Section 2.4 implies that for every path in a nondegenerate uniform adapted triangulation $T$ with consecutive vertices $v_{0} v_{1} \ldots v_{m}$ we have

$$
\begin{aligned}
E_{v_{0} v_{1} \ldots v_{m}}(u):=\sum_{k=1}^{m} c\left(\left[v_{k-1}, v_{k}\right]\right) \cdot\left(u\left(v_{k}\right)-u\left(v_{k-1}\right)\right)^{2} & \geqslant \sum_{k=1}^{m} \frac{\text { Const }}{e} \cdot\left(u\left(v_{k}\right)-u\left(v_{k-1}\right)\right)^{2} \\
& \geqslant \frac{\text { Const }}{e} \cdot \frac{1}{m}\left(\sum_{k=1}^{m} u\left(v_{k}\right)-u\left(v_{k-1}\right)\right)^{2} \\
& =\frac{\text { Const }}{e \cdot m}\left(u\left(v_{m}\right)-u\left(v_{0}\right)\right)^{2}
\end{aligned}
$$

Here $e$ denotes the eccentricity as defined in Section 4.1 and for the last estimate we have used Schwarz's inequality.

Now consider a simply connected triangulation $T^{\prime}$ with boundary contained in an open disc $B_{r}(v) \subset \mathbb{C}$. This is the assumption for part (i) of the lemma. In the case of part (ii), we consider the image triangulation $T_{g}^{\prime}$ by the chart $g_{O}(z)=(z-O)^{\gamma_{O}}$. By abuse of notation, we still denote this image triangulation by $T^{\prime}$. Also, we denote the vertices of $T^{\prime}$ by $Z$ and $W$, which are the actual vertices $z, w$ in the first case and 
the images $Z=g_{O}(z), W=g_{O}(w)$ in the case of a branch point. For simplicity, we assume that the edges between vertices are straight line segments, as we do not need the actual, possibly curved edges.

Let $u: V^{\prime} \rightarrow \mathbb{R}$ be any function which assumes its maximum and its minimum on the boundary for any subgraph of $T^{\prime}$. Let $Z, W$ be two distinct interior vertices of $T^{\prime}$. Denote by $Z W$ the straight line segment joining these points. Let $\operatorname{dist}\left(Z W, \partial T^{\prime}\right)$ be the Euclidean distance of this straight line segment to the curve of boundary edges. We assume that $|Z-W|<r / 3<\operatorname{dist}\left(Z W, \partial T^{\prime}\right) / 3$ for some $r>0$. Let $h^{\prime}$ denote twice the maximum circumradius of the triangles of $T^{\prime}$. Let $m=\left\lfloor\frac{r-|Z-W|}{2 h^{\prime}}\right\rfloor$ be the largest integer smaller than $\frac{r-|Z-W|}{2 h^{\prime}}$. We consider auxiliary rectangles $R_{k}, k=$ $1, \ldots, m$, which are centered at $(Z+W) / 2$ with one pair of sides parallel to $Z W$ with length $Z W+2 k \cdot h^{\prime}$ and other pair of sides orthogonal to $Z W$ of length $2 k \cdot h^{\prime}$. Then the interior of $R_{k}, k=1, \ldots, m$, is covered by triangles of $T^{\prime}$. Denote by $V_{k}^{\prime}$ the set of vertices contained in $R_{k} \backslash R_{k-1}$, where $R_{0}=Z W$. Then any two vertices $v_{A}, v_{B} \in V_{k}^{\prime}$ may be connected by a path $v_{0} v_{1} \ldots v_{N}$ with $v_{0}=v_{A}, v_{N}=v_{B}$ and all vertices $v_{j} \in V_{k}^{\prime}$ as $h^{\prime}$ is larger than any edge length.

Without loss of generality, assume that $u(Z) \geqslant u(W)$. As $u$ assumes its maximum and minimum on the boundary, there exists $Z_{k}, W_{k} \in V_{k}^{\prime}$ such that $u\left(Z_{k}\right) \geqslant u(Z) \geqslant$ $u(W) \geqslant u\left(W_{k}\right)$. The length of the path joining $Z_{k}$ and $W_{k}$ is at most the number of vertices in $V_{k}^{\prime}$. The set of these vertices can be covered by at most Const $\cdot(|Z-W|+$ $\left.4 k h^{\prime}\right) / h^{\prime}$ discs of radius $h^{\prime} / 2$. Therefore, by condition (U) of Section 2.4 the number of vertices in $V_{k}^{\prime}$ is less than Const $\cdot e \cdot\left(|Z-W| / h^{\prime}+4 k\right)$. Therefore, we can estimate the energy for a path $v_{0} v_{1} \ldots v_{N}$ in $V_{k}^{\prime}$ from $v_{0}=Z_{k}, v_{N}=W_{k}$ using (17)

$$
E_{Z_{k} \ldots W_{k}}(u) \geqslant \frac{\text { Const }}{e \cdot\left(\text { Const } \cdot \cdot\left(|Z-W| / h^{\prime}+4 k\right)\right)} \cdot\left(u\left(Z_{k}\right)-u\left(W_{k}\right)\right)^{2}=\text { Const } \cdot \frac{(u(Z)-u(W))^{2}}{e^{2}} \cdot \frac{h^{\prime}}{k h^{\prime}+|Z-W| / 4} .
$$

Summing these estimates and estimating $\sum_{k=1}^{m} \frac{h^{\prime}}{k h^{\prime}+|Z-W| / 4} \geqslant$ Const $\int_{h^{\prime}}^{\frac{r-|Z-W|}{2}} \frac{d t}{t+|Z-W| / 4}$ we get

$$
\begin{aligned}
E^{\prime}(u) & \geqslant \sum_{k=1}^{m} E_{Z_{k} \ldots W_{k}}(u) \geqslant \text { Const } \cdot \frac{(u(Z)-u(W))^{2}}{e^{2}} \cdot \int_{h^{\prime}}^{\frac{r-|Z-W|}{2}} \frac{d t}{t+|Z-W| / 4} \\
& \geqslant \text { Const } \cdot \frac{(u(Z)-u(W))^{2}}{e^{2}} \cdot \log \frac{2 r-|Z-W|}{4 h^{\prime}+|Z-W|} \\
& \geqslant \text { Const } \cdot \frac{(u(Z)-u(W))^{2}}{e^{2}} \cdot \log \frac{r}{3 \max \left\{|Z-W|, h^{\prime}\right\}} .
\end{aligned}
$$

This implies the desired inequalities (8) and (9).

Acknowledgements The authors especially thank Stefan Sechelmann for writing software and creating examples for numerical experiments.

Funding Open Access funding enabled and organized by Projekt DEAL. This research was supported by the DFG Collaborative Research Center TRR 109 "Discretization in Geometry and Dynamics". 


\section{Declarations}

Conflict of Interests There are no conflicts of interest.

Open Access This article is licensed under a Creative Commons Attribution 4.0 International License, which permits use, sharing, adaptation, distribution and reproduction in any medium or format, as long as you give appropriate credit to the original author(s) and the source, provide a link to the Creative Commons licence, and indicate if changes were made. The images or other third party material in this article are included in the article's Creative Commons licence, unless indicated otherwise in a credit line to the material. If material is not included in the article's Creative Commons licence and your intended use is not permitted by statutory regulation or exceeds the permitted use, you will need to obtain permission directly from the copyright holder. To view a copy of this licence, visit http://creativecommons.org/licenses/by/4.0/.

\section{References}

1. Bobenko, A., Pinkall, U., Springborn, B.: Discrete conformal maps and ideal hyperbolic polyhedra. Geom. Topol. 19(4), 2155-2215 (2015)

2. Bobenko, A.I., Günther, F.: Discrete complex analysis on planar quad-graphs. In: Advances in Discrete Differential Geometry, pp. 57-132. Springer, Berlin (2016)

3. Bobenko, A.I., Günther, F.: Discrete Riemann surfaces based on quadrilateral cellular decompositions. Adv. Math. 311, 885-932 (2017)

4. Bobenko, A.I., Mercat, C., Schmies, M.: Period matrices of polyhedral surfaces. In: Bobenko, A.I., Klein, C. (eds.) Computational Approach to Riemann Surfaces, pp. 213-226. Springer (2011)

5. Bobenko, A.I., Mercat, C., Suris, Y.B.: Linear and nonlinear theories of discrete analytic functions. Integrable structure and isomonodromic Green's function. J. reine angew. Math. 583, 117-161 (2005)

6. Bobenko, A.I., Pinkall, U.: Discrete isothermic surfaces. J. Reine Angew. Math. 475, 187-208 (1996)

7. Bobenko, A.I., Skopenkov, M.: Discrete Riemann surfaces: linear discretization and its convergence. J. Reine Angew. Math. 720, 217-250 (2016)

8. Bobenko, A.I., Springborn, B.A.: Variational principles for circle patterns and Koebe's theorem. Trans. Amer. Math. Soc. 356, 659-689 (2004)

9. Bücking, U.: Approximation of conformal mappings by circle patterns. Geom. Dedicata 137, 163-197 (2008)

10. Bücking, U.: Approximation of conformal mappings on triangular lattices. In: Bobenko, A. (ed.) Advances in Discrete Differential Geometry. Springer (2016)

11. Chelkak, D., Smirnov, S.: Discrete complex analysis on isoradial graphs. Adv. in Math. 228(3), 15901630 (2011)

12. Courant, R., Friedrichs, K., Lewy, H.: Über die partiellen Differentialgleichungen der mathematischen Physik. Math. Ann. 100, 32-74 (1928)

13. Deconinck, B., van Hoeij, M.: Computing Riemann matrices of algebraic curves. Physica D 152-153, 28-46 (2001). Advances in Nonlinear Mathematics and Science: A Special Issue to Honor Vladimir Zakharov

14. Duffin, R.J.: Discrete potential theory. Duke Math. J. 20, 233-251 (1953)

15. Duffin, R.J.: Basic properties of discrete analytic functions. Duke Math. J. 23, 335-363 (1956)

16. Duffin, R.J.: Distributed and lumped networks. J. Math. Mech. 8, 793-826 (1959)

17. Duffin, R.J.: Potential theory on a rhombic lattice. J. Combin. Th. 5, 258-272 (1968)

18. Dynnikov, I., Novikov, S.: Geometry of the triangle equation on two-manifolds. Mosc. Math. J. 3, 419-438 (2003)

19. Ferrand, J.: Fonctions préharmoniques et fonctions préholomorphes. Bull. Sci. Math. 68, 152-180 (1944)

20. Frauendiener, J., Klein, C.: Computational approach to hyperelliptic Riemann surfaces. Lett. Math. Phys. 105(3), 379-400 (2015)

21. Frauendiener, J., Klein, C.: Computational approach to compact Riemann surfaces. Nonlinearity 30(1), 138-172 (2017)

22. Gianni, P., Seppälä, M., Silhol, R., Trager, B.: Riemann surfaces, plane algebraic curves and their period matrices. J. Symbolic Comput. 26(6), 789-803 (1998) 
23. Isaacs, R.P.: A finite difference function theory. Univ. Nac. Tucumá,n Revista A 2, 177-201 (1941)

24. Lazarus, F., Pocchiola, M., Vegter, G., Verroust, A.: Computing a canonical polygonal schema of an orientable triangulated surface. In: Proceedings of the Seventeenth Annual Symposium on Computational Geometry, SCG '01, pp. 80-89, Association for Computing Machinery, New York, NY, USA (2001)

25. Lelong-Ferrand, J.: Représentation confrome et transformations à intégrale de Dirichlet bornée. Gauthier-Villars, Paris (1955)

26. Lusternik, L.: Über einige Anwendunge der direkten Methoden in der Variationsrechnung. Mat. Sb. 33(2), 173-201 (1926)

27. MacNeal, R.: The solution of partial differential equations by means of electrical networks. Ph.D. thesis California Institute of Technology (1949)

28. Matthes, D.: Convergence in discrete Cauchy problems and applications to circle patterns. Conform. Geom. Dyn. 9, 1-23 (2005)

29. Mercat, C.: Discrete Riemann surfaces and the Ising model. Commun. Math. Phys. 218, 177-216 (2001)

30. Mercat, C.: Discrete period matrices and related topics. arXiv:math-ph/0111043 (2002)

31. Mercat, C.: Discrete Riemann surfaces. In: Papadopoulos, A. (ed.) Handbook of Teichmüller Theory, IRMA Lectures in Mathematics and Theoretical Physics, vol. 11, pp. 541-575 (2007). Eur. Math. Soc.

32. Meyer, M., Desbrun, M., Schröder, P., Barr, A.H.: Discrete differential geometry operators for triangulated 2-manifolds. In: Visualization and Mathematics III, pp. 35-57. Springer (2003)

33. Molin, P., Neurohr, C.: Computing period matrices and the Abel-Jacobi map of superelliptic curves. arXiv:1707.07249 [math.NT] (2017)

34. Novikov, S.: New discretization of complex analysis: the Euclidean and Hyperbolic planes. Proc Steklov Inst Math 273, 238-251 (2011)

35. Pinkall, U., Polthier, K.: Computing discrete minimal surfaces and their conjugates. Experiment. Math. 2, 15-36 (1993)

36. Schramm, O.: Circle patterns with the combinatorics of the square grid. Duke Math. J. 86, 347-389 (1997)

37. Skopenkov, M.: The boundary value problem for discrete analytic functions. Adv. Math. 240, 61-87 (2013)

38. Stephenson, K.: Introduction to circle packing: the theory of discrete analytic functions. Cambridge University Press, New York (2005)

39. Zeng, W., Jin, M., Luo, F., Gu, X.: Canonical homotopy class representative using hyperbolic structure. 171-178 (2009)

Publisher's Note Springer Nature remains neutral with regard to jurisdictional claims in published maps and institutional affiliations. 